\title{
A Comparison of Linear and Branched Wormlike Micelles using Large Amplitude Oscillatory Shear and Orthogonal Superposition Rheology
}

\author{
Sunilkumar Khandavalli ${ }^{1}$, Jan Hendricks ${ }^{2}$ \\ Christian Clasen ${ }^{2}$ and Jonathan P. Rothstein ${ }^{1}$ \\ ${ }^{1}$ Mechanical and Industrial Engineering Department, University of Massachusetts - Amherst, USA \\ ${ }^{2}$ Department of Chemical Engineering, University of Leuven, KU Leuven, Belgium
}

\begin{abstract}
In this study, we present a comparative investigation of linear and branched wormlike micelles using two non-linear rheological tools; orthogonal superposition rheology and large amplitude oscillatory shear rheology (LAOS). The surfactants were a series of mixtures of octyl trimethyl ammonium bromide $\left(\mathrm{C}_{8} \mathrm{TAB}\right)$ and sodium oleate $(\mathrm{NaOA})$. A transition from linear to branched wormlike micelles was obtained by either varying the relative ratio of $\mathrm{NaOA}$ to $\mathrm{C}_{8} \mathrm{TAB}$ at a fixed total surfactant concentration or by fixing the ratio of $\mathrm{NaOA}$ to $\mathrm{C}_{8} \mathrm{TAB}$ and varying the total surfactant concentration. Orthogonal superposition rheology imposes a small amplitude oscillatory shear strain over an orthogonally-imposed shear flow to probe the effect of shear on the storage and loss modulus of the fluid. For both the linear and branched wormlike micelle solutions, the plateau modulus, the relaxation times and the dynamic viscosity were all found to be sensitive to the strength of the orthogonally-imposed shear rate. However, the nonlinear effects were much more pronounced for the case of the branched wormlike micelle solutions. This is likely due to a break-down of the branched wormlike micelles under flow which can be inferred from the decrease in the plateau modulus and the subsequent increase in the calculated mesh size of the entangled micelle network with increasing orthogonal shear-rate. In the LAOS measurements, both the linear and branched wormlike micelles exhibited a qualitatively similar trend in the viscoelastic nonlinearities as the strain amplitude of the imposed oscillatory flow was increased. However, the strength of viscoelastic non-linearities, both within an oscillatory cycle and with increasing strain amplitude, of the branched wormlike micelles were found to be significantly larger than those observed for the linear wormlike micelles. Additionally, at large strain amplitudes, the Lissajous-Bowditch plots of the branched wormlike micelle solutions were found to exhibit a secondary loop resulting from a negative elastic modulus. This is indicative of a stress overshoot and likely the result of a cyclical breakdown and reformation of the underlying wormlike micelle entangled structure during an oscillatory cycle.
\end{abstract}




\section{Introduction}

The high volume of publications in the recent rheology literature on the topic of nonlinear viscoelasticity measurements has demonstrated the desire by the broader rheological community for new experimental techniques to measure the nonlinear response of complex fluids and the need for clarity in the physical interpretation of these measurements. Here we will focus on two different nonlinear shear rheology techniques, large amplitude oscillatory shear (LAOS) [1-3] and Orthogonal Superposition Rheology $[4,5]$. These techniques will be applied to a series of wormlike micelle solutions with the goal

of discovering a nonlinear rheological fingerprint that can distinguish between linear and branched wormlike micelles. In the processes, these two very different techniques will be directly compared and contrasted with the pros and cons of each explored in some detail.

Both LAOS and orthogonal superposition utilize oscillatory shear flows over a wide range of frequencies to probe the relaxation modes of the complex fluids. Both techniques allow for the measurement of storage and loss moduli (or the dynamic viscosity). The main difference in these two techniques is how the transition from the linear to the nonlinear response of the material is achieved. In orthogonal superposition rheology, a small amplitude oscillatory shear (SAOS) flow is superimposed upon a steady shear flow perpendicular to the direction of the shear flow. As these two shear flows are orthogonal, the response can be decoupled (in contrast to parallel superimposed oscillatory shear [6]), and, as a result, the data can be interpreted using standard SAOS rheological material functions to understand the response of rheologically complex fluids and the variation of the fluid's microstructure under a non-linear steady shear flow. In LAOS, the nonlinearity is achieved by increasing the amplitude of the imposed sinusoidal oscillations. The resulting flow is more complicated with the shear rate and strain varying throughout the oscillatory cycle and a new set of rheological functions is needed to interpret the results. The advantage of LAOS (and complementarity to OSP) is that the transition from linear to nonlinear response can be observed for the resting sample without an alteration of the underlying structure of the fluid.

\subsection{Orthogonal Superposition Rheology}

Orthogonal superposition measurements have received little attention compared to the recent emphasis on the literature on LAOS. This is primarily the result of the technical complexity of implementing an orthogonal superposition experiment. Although parallel superposition measurements and LAOS are currently available on commercial shear rheometers, until very recently orthogonal superposition measurements were only accessible using home-built devices which utilize the force rebalancing normal force transducer to impose a vertical oscillation on top of rotational shear flow in a 
double wall Couette cell like the one shown schematically in Figure 1 [5]. In addition, due to the limitations in the range of the normal force transducers, these measurements are limited to fluids with relatively high viscosities.

In orthogonal superposition, a steady rotational shear flow is introduced using a double wall Couette such that

$$
\gamma_{\|}=\gamma_{r \theta}=\text { constant. }
$$

Superimposed upon this steady shear is a small amplitude oscillatory shear flow along the vertical axis of the Couette cell such that

$$
\gamma_{\perp}=\gamma_{r z}=\gamma_{0} \omega \cos \left(\omega_{1} t\right)
$$

where $\gamma_{0}$ and $\omega_{1}$ are input strain amplitude and frequency respectively. The resulting oscillating force has the same frequency as the applied shear field, but with a phase shift. In Vermant et al. [5] they demonstrated how the measured force signal could be converted into a storage and loss modulus, $G$ ' and $G$ ', with knowledge of the flow geometry and proper calibration of the instrument to account for the mass of the fixture, the spring constant of the instrument and the friction coefficient of the force transduce. Thus using orthogonal superposition rheometry, the linear viscoelastic response of the fluid can be probed in one direction as a constant, nonlinear steady shear flow is applied in an orthogonal direction.

\subsection{Large amplitude oscillatory shear (LAOS)}

Large amplitude oscillatory shear flow is an extension of small amplitude oscillatory shear to larger strains. In dynamic oscillatory shear rheology, a sinusoidal strain signal is imposed on a material given as,

$$
\gamma(t)=\gamma_{0} \sin \left(\omega_{1} t\right)
$$

where $\gamma_{0}$ and $\omega_{1}$ are input strain amplitude and frequency respectively. For small strain amplitudes, the resulting stress response is also sinusoidal. The linear viscoelastic response is represented as

$$
\sigma(t)=\gamma_{0}\left[G_{1}^{\prime}\left(\omega_{1}\right) \sin \left(\omega_{1} t\right)+G_{1}^{\prime \prime}\left(\omega_{1}\right) \cos \left(\omega_{1} t\right)\right]
$$

where the in-phase and out-of-phase components of the stress response are termed as storage modulus, $G_{1}^{\prime}$, and loss modulus, $G_{1}^{\prime \prime}$. When the input strain amplitude is increased beyond a critical value, the stress response becomes nonlinear and can be represented as

$$
\sigma(t, \gamma, \omega)=\sum \sigma_{n} \sin \left(n \omega_{1} t+\delta_{n}\right)
$$


where additional higher harmonic stress components $(n>1)$ are present in the stress response. Therefore, the moduli $G_{1}^{\prime}$ and $G_{1}^{\prime \prime}$, which are based on the fundamental frequency, do not fully describe the mechanical behavior beyond the linear viscoelastic limit and the higher harmonics cannot be ignored. One common method to examine a nonlinear response is by performing a Fourier transform and examining the frequency spectrum [7, 8]. The intensity of higher stress harmonics are an indication of the degree of nonlinearity. This method has been a valuable tool for characterizing nonlinear behavior for several materials such as, polymer melts, filled rubbers [9, 10], suspensions and emulsions [11-14]. Only odd harmonics appear in the nonlinear region, and the appearance is assumed due to odd symmetry with respect to the directionality of shear strain [15]. The appearance of even harmonics in the Fourier spectrum can be attributed to the presence of secondary flows [16], asymmetric wall slip or edge effects [17]. However, Fourier transform rheology lacks a direct physical interpretation of the material response. Another common method for investigating the nonlinear response is to follow a graphical approach, where stress versus strain curves known as Lissajous-Bowditch curves are studied. The nonlinear behavior of the material can be identified from the shape of the Lissajous-Bowditch or stress versus strain curve, as follows: linear - purely elastic; circle - purely viscous; ellipse - linear viscoelastic and distorted ellipse - nonlinear viscoelastic. Thus, the progressive transition from linear to nonlinear viscoelastic nature of the material with strain amplitude can be studied from the graphical representation. The total stress can further be decomposed into elastic and viscous components through simple algebraic manipulations using the even and odd nature of the trigonometric functions [18]. Extending Cho's stress decomposition method [18], Ewoldt et al. [2] proposed more physically meaningful nonlinear viscoelastic measures by representing elastic and viscous stresses through orthogonal Chebyshev polynomials of the first kind. Based on this, Ewoldt et al. [2] also defined nonlinear viscoelastic moduli based on the local material response at small and large instantaneous strains/strain-rates in an oscillatory cycle [2]. The nonlinear elastic material parameters are given in terms of Fourier coefficients as,

$$
\begin{gathered}
G_{M}^{\prime}=\left.\frac{d \sigma}{d \gamma}\right|_{\gamma=0}=\sum_{n=o d d} n G_{n}^{\prime}, \\
G_{L}^{\prime}=\left.\frac{\sigma}{\gamma}\right|_{\gamma= \pm \gamma_{0}}=\sum_{n=o d d} G_{n}^{\prime}(-1)^{(n-1) / 2},
\end{gathered}
$$

where $G_{M}^{\prime}$ is the dynamic modulus measured at minimum strain and $G_{L}^{\prime}$ is the large strain dynamic modulus. These measures can be obtained from Fourier coefficients or from numerical differentiation of the material response. In the linear-viscoelastic regime, these measures reduce to first harmonic moduli, $G_{M}^{\prime}=G_{L}^{\prime}=G_{1}^{\prime}\left(\omega_{0}\right)$ 
Similarly the corresponding nonlinear viscous material properties are given as,

$$
\begin{gathered}
\eta_{M}^{\prime}=\left.\frac{d \sigma}{d \gamma}\right|_{\gamma=0}=\frac{1}{\omega_{n}} \sum_{n=\text { odd }} n G_{n}^{\prime \prime}(-1)^{(n-1) / 2}, \\
\eta_{L}^{\prime}=\left.\frac{\sigma}{\gamma}\right|_{\gamma= \pm \gamma_{0}}=\frac{1}{\omega_{n}} \sum_{n=\text { odd }} G_{n}^{\prime \prime}
\end{gathered}
$$

where $\eta_{M}^{\prime}$ is the dynamic viscosity measured at minimum strain-rate and $\eta_{L}^{\prime}$ is the dynamic viscosity measured at maximum strain-rate. From these measures, the dominant intra-cycle viscoelastic nonlinearities can also be captured with a single descriptor. The elastic nonlinearity is quantified as the strain-stiffening ratio, $S$, given by

$$
S=\frac{G_{L}^{\prime}-G_{M}^{\prime}}{G_{L}^{\prime}} .
$$

The intra-cycle elastic nonlinearity is strain-stiffening when $S>0$ and is strain-softening when $S<0$. The corresponding intra-cycle viscous nonlinearity, quantified as shear-thickening ratio, $T$, is given by,

$$
T=\frac{\eta_{L}^{\prime}-\eta_{M}^{\prime}}{\eta_{L}^{\prime}} .
$$

For the case when $T>0$ the behavior is intra-cycle shear-thickening and is intra-cycle shear-thinning when $T<0$. A detailed description of the nonlinear viscoelastic measures can be found in (Hyun et al. 2011; Ewoldt et al. 2008).

In addition to studying the nonlinearity within an oscillatory cycle (intra-cycle) through the local nonlinear viscoelastic moduli described above, further insights into the origins of the observed nonlinearity can be gained by examining the change in the viscoelastic nonlinear material properties with increasing imposed strain amplitude (inter-cycle) [2]. Within an oscillatory cycle, the strain and strain rate vary orthogonally. As a result, as the strain is maximized within a cycle, the strain rate goes to zero and vice versa. Thus the nonlinear viscoelastic response within a given cycle can be affected by the varying strain and/or the varying strain-rate. For the case of elastic nonlinearities, the variation of the large-strain modulus, $G_{L}^{\prime}$, with increasing imposed strain amplitude can be attributed to strain-induced nonlinearities since the strain rate at maximum strain is zero. Similarly, the variation of the corresponding minimum-strain modulus, $G_{M}^{\prime}$, with increasing imposed strain-amplitude can be attributed to strain-rate induced nonlinearities as the modulus is evaluated at the point of zero strain within an oscillatory cycle. Thus changes to $G_{L}^{\prime}$ can be thought of as strain softening or strain hardening while 
changes to $G_{M}^{\prime}$ can be thought of as strain-rate softening or strain-rate hardening. By comparing the relative magnitude of $G_{M}^{\prime}$ and $G_{L}^{\prime}$, as a function of strain amplitude, one can infer whether the observed nonlinearities are dominated by changes in strain or strain rate. As a consequence, when the magnitude of $G_{M}^{\prime} \gg G_{L}^{\prime}$ we will designate the nonlinear response of the fluid to be strain-rate hardening/softening while if the magnitude $G_{M}^{\prime} \ll<G_{L}^{\prime}$ we will designate the fluid's nonlinear response to be strain hardening/softening. In a similar manner, the dependence of the viscous nonlinearity on the amplitude of the imposed strain or strain-rate within an oscillatory cycle, can be studied by examining the inter-cycle variation of the local nonlinear dynamic viscosity measures, $\eta_{M}^{\prime}$ and $\eta_{L}^{\prime}$. As before, since $\eta_{L}^{\prime}$ is evaluated at the maximum strain rate and $\eta_{M}^{\prime}$ is evaluated when the strain rate is zero and the strain is maximized, changes to $\eta_{L}^{\prime}$ can be thought of as strain-rate thinning or strain-rate thickening, while changes to $\eta_{M}^{\prime}$ can be thought of as strain thinning or strain thickening. If the magnitude of $\eta_{L}^{\prime} \gg \eta_{M}^{\prime}$, then the viscous nonlinearities can be considered dominated by strain-rate thickening/thinning effect while if the magnitude of $\eta_{L}^{\prime}<<\eta_{M}^{\prime}$ then the viscous nonlinearities can be thought of as dominated by strain thickening/thinning effects.

It should be noted that this softening, hardening, thickening and thinning is not the response of the intrinsic sample at rest to the rate or deformation, but rather the response of the microstructural state that the sample takes on under this quasi-steady non-linear deformation.

\subsection{Wormlike Micelle Solutions}

Surfactants are amphiphilic molecules which have both a hydrophilic head which is often charged and a relatively short hydrophobic tail. When the surfactant molecules are dissolved in water above their critical micelle concentration (CMC), they can spontaneously self-assemble into large aggregates known as micelles [19-22]. These large aggregates can form a number of different complex shapes depending on the size of the surfactant head group, the length and number of tails, the charge on the surfactant, the salinity of the solution, and the flow conditions [19]. A considerable amount of research has been performed over the past few years to investigate the morphology, the phase transitions, and the rheology of different surfactant solutions [21,23-26]. Of interest to us are surfactants which tend to form wormlike micelles because at large enough surfactant concentration these wormlike micelles can grow very long, become flexible and entangle thereby making the solution viscoelastic much like polymer solutions.

Although both wormlike micelle solutions and polymer solutions can be viscoelastic, wormlike micelles are physically quite different from polymers. Whereas the backbone of a polymer is covalently 
bonded and rigid, wormlike micelles are held together by relatively-weak physical attractions and as a result are continuously breaking and reforming with time. In an entangled network, both individual polymer chains and wormlike micelles can relief stress through reptation driven by Brownian motion [20]. However, unlike polymeric fluids, wormlike micelle solutions have access to a number of stress relief mechanisms in addition to reptation. Wormlike micelles can also relieve stress and eliminate entanglement points by either breaking and reforming in a lower stress state [21] or alternatively by creating temporary branch point which allows two entangled micelles to pull right through each other in thereby eliminating the entanglement point and relieving stress in what has become known as a 'ghostlike' crossing [27]. The reptation and breakup relaxation mechanisms have characteristic time scales of $\lambda_{\text {rep }}$ and $\lambda_{b r}$ respectively.

Lequeux and Candau [28] demonstrated that the phase diagram of surfactant solutions that form wormlike micelle solutions can be quite complex. Within the semi-dilute regime, increasing salt concentration can drive the wormlike micelles from linear, to branched and finally to an interconnected saturated network. For a linear wormlike micelle, the shape and area per unit surfactant molecule is optimized at all positions along the backbone except at the ends [19]. When a linear micelle breaks it must pay an energy penalty by forming two new end caps. In this regime, the electrostatic repulsion of the head groups is strong enough that the increased curvature of an endcap which spreads the head groups apart is favored over the concave curvature of a branch point which drives the charged head groups of the surfactants closer together. However, as the salt concentration is increased and the head group charges are sufficiently screened, the wormlike micelles can form three-point or four-point junctions. Evidence of the existence of branched micelles can be seen in Cryo-TEM images [29, 30], inferred directly from Pulse Gradient Spin-Echo nuclear magnetic resonance (PGSE NMR) measurements [31, 32] and predicted by molecular dynamics simulations [33].

A number of branched wormlike micelle systems have been developed recently and their shear rheology has been well characterized [34-39]. In the experiments described in this manuscript, we have chosen to focus on the mixed anionic and cationic surfactants pioneered by Raghavan and Kaler [37, 38] because the systems they developed have viscosities and relaxation times suitable for both LAOS and for Orthogonal Superposition Rheology (OSR). Specifically, Raghavan et al. [38] used a series of mixtures of octyl trimethyl ammonium bromide $\left(\mathrm{C}_{8} \mathrm{TAB}\right)$ and sodium oleate $(\mathrm{NaOA})$ to obtain both linear and branched wormlike micelles by both varying the relative ratio of $\mathrm{NaOA}$ to $\mathrm{C}_{8} \mathrm{TAB}$ or by fixing the ratio of $\mathrm{NaOA}$ to $\mathrm{C}_{8} \mathrm{TAB}$ and varying the total surfactant concentration. At a fixed ratio of $\mathrm{NaOA} / \mathrm{C}_{8} \mathrm{TAB}$ of $70 \% / 30 \%$, their shear rheology measurements showed a maximum in the shear viscosity at $4 \mathrm{wt} \%$ while the elastic modulus of the fluids increased monotonically with increasing surfactant concentrations. Due 
to the presence of this maximum in these and a number of other wormlike micelle solutions, solutions with very different compositions can have identical rheological properties in shear flow curves. Raghavan et al. [38] hypothesized, and later demonstrated through cryo-TEM imaging, that the maximum in the shear viscosity is due to the transition from linear to branched micelles [30, 40]. Recently, the negative effect of branching on shear viscosity has been confirmed by molecular dynamics simulations [33]. For these systems, branching is achieved by adequately screening the surfactant head groups either through a stoichiometric balance of the oppositely charged surfactant headgroups along the micelle or alternatively through the release of surfactant counterions which results in a reduction of the electrostatic double layer around the micelle [38]. A similar reduction in the shear viscosity has been observed for reversed micelle systems with the onset of branching [31,32]. In the experiments of Palazzo et al. [31, 32], a series of reverse micelle solutions comprised of lecithin, water and oil were studied with both shear rheology and PGSE-NMR diffusion measurements which were able to directly confirm the presence of micellar branching.

The decrease in the shear viscosity can be attributed to a new stress relief mechanics applicable only to branched micelles whereby the branch points are not fixed at a specific point along the backbone as is the case for branched polymers, but are free to slide along the micelle resulting in an additional stress relaxation mode not accessible in linear systems [36, 41]. The branched points formed in wormlike micelles and their effect on the shear rheology are very different from branched points in polymers which because they are fixed along the polymer backbone make movement through reptation more difficult and can dramatically increase the relaxation time and elasticity of the polymeric fluids [15].

One place where rheological differences between branching in polymeric liquids and wormlike micelle solutions has been recently observed is in extensional rheology measurements. In extensional flows of linear and long-chain-branched polyolefin melts with similar shear rheology, the branched polymers were found to exhibit significantly enhanced strain hardening in transient extensional flows [42, 43]. The increase in extensional viscosity is a result of increased chain stretching and reptation tube dilation resulting from the relative difficulty associated with moving a branched point in flow. For wormlike micelle solutions, the opposite trend has been observed because as described above, the branch point does not represent a similar hindrance to flow. It has been shown recently that the presence of branching in wormlike micelle solutions can reduce and nearly eliminate strain hardening of the extensional viscosity with Trouton ratio approaching the Newtonian limit of three, $\operatorname{Tr}=\eta_{E} / \eta=3[44$, 45]. In comparison to this, linear wormlike micelle solutions can show Trouton ratios approaching $T r=$ 1000 [26, 44-49]. In this manuscript we will investigate whether other nonlinear rheological measurements can make similar distinctions between linear and branched wormlike micelle systems. 


\section{Experimental}

\subsection{Materials}

A series of wormlike micelle solutions were prepared with a total surfactant concentrations ranging from $3 \mathrm{wt} \%$ to $6 \mathrm{wt} \%$ by dissolving the surfactants octyl trimethyl ammonium bromide $\left(\mathrm{C}_{8} \mathrm{TAB}\right)$ and sodium oleate $(\mathrm{NaOA})$ in deionized water. For the initial study presented here, the ratio of the two surfactants $\mathrm{NaOA} / \mathrm{C}_{8} \mathrm{TAB}$ was fixed at $70 / 30$ and the total concentration was varied from $3 \mathrm{wt} \%$ to $6 \mathrm{wt} \%$. At this surfactant ratio, the solutions have been shown to transition from linear to increasingly branched micelles as the concentration was increased beyond $4 \mathrm{wt} \%[30,40]$, so that the $3 \mathrm{wt} \% 70 / 30 \mathrm{NaOA} / \mathrm{C}_{8} \mathrm{TAB}$ solution represents linear micelles, whereas the $4.5 \mathrm{wt} \%$ and $6 \mathrm{wt} \% 70 / 30 \mathrm{NaOA} / \mathrm{C}_{8} \mathrm{TAB}$ both represent branched micelles. For the second study presented here, the total concentration of the surfactants was fixed at $6 \mathrm{wt} \%$, but the ratio of $\mathrm{NaOA} / \mathrm{C}_{8} \mathrm{TAB}$ was increased from $70 / 30$ to $85 / 15$. A similar transition from branched to linear wormlike micelles was also observed as the $\mathrm{C}_{8} \mathrm{TAB}$ portion in the ratio was decreased from 30 to $15 \%$, so that the $6 \mathrm{wt} \% 85 / 15 \mathrm{NaOA} / \mathrm{C}_{8} \mathrm{TAB}$ represents also a linear micelle solution. To speed dissolution and insure a homogeneous mixture, the surfactant solutions were mixed with a magnetic stirring bar for up to 36 hours. After the solutions were fully dissolved, they were allowed to settle at room temperature for at least 24 hours before any experiments were performed to allow the air bubbles entrained during stirring to leave the sample. All shear experiments were performed at a temperature of $T=20^{\circ} \mathrm{C}$.

\subsection{Shear Rheometry}

The Large Amplitude Oscillatory shear rheology was conducted on a stress-controlled TA DHR-3 rheometer using a $40 \mathrm{~mm} 2^{\circ}$ aluminum cone-plate geometry and a solvent trap at a constant temperature of $20^{\circ} \mathrm{C}$. The orthogonal superposition measurement were performed using a double wall Couette cell on a TA ARES G2 specially modified for the measurements [4] at a constant temperature of $20^{\circ} \mathrm{C}$. Steady shear rheological measurements were conducted on both rheometers in the shear rate range of 0.001 to $100 \mathrm{~s}^{-1}$. The small amplitude oscillatory shear tests were conducted on both rheometers in the frequency range of 0.001 to $100 \mathrm{rad} / \mathrm{s}$, with a fixed strain amplitude chosen to place the measurements well within the linear viscoelastic region.

The LAOS measurements were conducted for a strain amplitude range between 0.01 and $1000 \%$ for a frequency of $3 \mathrm{rad} / \mathrm{s}$. A sufficient number of oscillatory cycles were run, ranging from 30 to 80 to ensure a steady-state measurement. The nonlinear viscoelastic material parameters such as, the LissajousBowditch plots and the nonlinear viscoelastic parameters such as minimum/maximum-strain dynamic moduli and minimum/maximum-rate dynamic viscosity, were obtained using the Fourier-transform 
software application of the TA DHR-3 rheometer. The FT-Rheology application processes the raw torque signal and extracts nonlinear parameters from the reconstructed/filtered signal. The reconstructed stress signal was double checked with the steady raw stress signal to check for poor reconstruction of any noisy signal. The data points affected by noise or inaccurate analysis of TA's TRIOS software were either excluded from the manuscript or the nonlinear viscoelastic parameters were recalculated from the Lissajous-Bowditch plots. As the LAOS measurements were performed on a stress controlled rheometer, the data can be affected by inertia [50]. An oscillatory mapping of the geometry was performed to compensate for the influence of instrument inertia. However, when the torque generated by instrument inertia is large, the rheometer's compensation routines are not sufficient to completely eliminate the effect of instrument inertia. To insure high quality data, the relative ratio of inertia torque to sample torque, $T_{\text {inertia }} / T_{\text {sample }}$, was checked for each experimental data point and was found to be very low, $T_{\text {inertia }} / T_{\text {sample }}$ $<5 \%$, indicating the influence of inertia on the sample data is very small [50]. Any experimental artifacts such as wall slip and edge fracture in measurements are identified by examining the presence of even harmonic intensity which are sensitive to asymmetric artifacts [51]. For all cases, the magnitude of even harmonics were found to be very small.

The orthogonal superposition measurements were performed utilizing the force rebalanced transducer of the ARES-G2 rotational rheometer. The control loop of the normal force transducer has been modified to generate an axial motion, orthogonal to the standard angular motion of the rheometer. To create the uniform oscillatory deformation orthogonal to the main flow direction, a double-wall Couette geometry has been used (Figure 1). The sinusoidal shear flow orthogonal to the main flows is obtained by moving a hollow bob axially, whereas the tangential steady shear flow is achieved by rotating the double-walled Couette cup. To minimize pumping flow effects during orthogonal superposition measurements, an opening is made in the inner wall of the cup that connects with a liquid reservoir in the center of the cup [4].

Small amplitude oscillatory shear measurements were performed with zero applied rotation shear rate both in the $\theta$ and the $z$ directions (parallel and orthogonal to the rotational shear direction). The results of these two SAOS measurements were compared to insure that the orthogonal measurements were accurate and that no anisotropy was introduced during loading of the Couette cell. Strain amplitude sweeps in both the $\theta$ and $z$ direction were performed to insure a linear response of the wormlike micelle solutions. In all cases, the applied strain of the z-direction oscillations was less than $5 \%$ which was well within the linear regime. Subsequently orthogonal oscillations in the $z$ direction with a variable frequency $\omega$ were superposed onto steady rotational shear rates between $0<\gamma_{r \theta}<10 \mathrm{~s}^{-1}$ 


\section{Results and Discussion}

\subsection{Steady shear rheology}

The steady-shear rheology of the four wormlike micelle solutions is presented in Figure 2. All four samples display a strong shear thinning of the viscosity. For all the fluids that were tested, the shear thinning response is quite similar. In each case, the slope of the decay in viscosity approaches, but does not reach $\eta \propto \gamma^{-1}$, which would be indicative of shear banding. Thus, although steady shear measurements are a nonlinear probe of the fluids, they are not an effective technique for differentiating between linear and branched micelle solutions.

\subsection{Small amplitude oscillatory shear rheology (SAOS)}

In Figure 3, the small amplitude oscillatory shear rheology is compared for all four $\mathrm{NaOA} / \mathrm{C}_{8} \mathrm{TAB}$ wormlike micelle solutions tested by plotting the storage modulus, $G^{\prime}$, and loss modulus, $G^{\prime \prime}$, as a function of angular frequency, $\omega$. For the fluids $70 / 30$ tested $\mathrm{NaOA} / \mathrm{C}_{8} \mathrm{TAB}$, the linear viscoelastic data can be fitted well with a one or two mode Maxwell model

$$
\begin{aligned}
G^{\prime} & =\sum_{i=1}^{n} \frac{\eta_{i} \lambda_{i} \omega^{2}}{1+\left(\lambda_{i} \omega\right)^{2}}, \\
G^{\prime \prime} & =\sum_{i=1}^{n} \frac{\eta_{i} \omega}{1+\left(\lambda_{i} \omega\right)^{2}},
\end{aligned}
$$

where $G^{\prime}$ and $G^{\prime \prime}$ are the loss and storage moduli respectively, $\lambda_{i}$ are the discrete relaxation times, $\eta_{i}$ are the discrete viscosities and $\omega$ is the angular velocity. Dividing the viscosity by the relaxation time we get the modulus of each mode of the fluid, $G_{i}=\eta_{i} / \lambda_{i}$. In the fast-breaking limit, where the break-up time is much shorter than the reptation time, $\lambda_{b r}<<\lambda_{\text {rep }}$, Cates' model [52] predicts that the linear viscoelastic response of these wormlike micelle solutions can be described by Maxwell fluids with a single relaxation time that is the geometric mean of the reptation and break-up time, $\lambda=\left(\lambda_{\text {rep }} \lambda_{b r}\right)^{1 / 2}$. The deviation of the rheological data from the predictions of the single mode Maxwell model at large angular frequencies in Figure 3 corresponds to the Rouse-like behavior of the micelle between entanglement points [53]. Kern et al. [54] showed the break-up time of the wormlike micelles, $\lambda_{b r}$, roughly corresponds to the angular frequency at which the data deviates from the predictions of the single-mode Maxwell model.

The zero shear viscosity, $\eta_{0}$, and the plateau modulus, $G_{0}$, derived from a single mode Maxwell model fit are tabulated in Table 1 along with the approximate value of the breakup time, $\lambda_{b r}$. The shear rheology is in good agreement with the values of shear rheology presented in the literature for identical 
systems $[38,44]$. A maximum in the viscosity data with increasing concentration is observed at $4.5 \mathrm{wt} \%$ after which the viscosity begins to decrease with increasing total surfactant concentration. Similarly, literature shows a similar maximum for a total concentration of $6 \mathrm{wt} \%$ surfactant for a relative concentration of $80 / 20 \mathrm{NaOA} / \mathrm{C}_{8} \mathrm{TAB}$ [38]. It should be noted, that this maximum in viscosity, which is due to the transition from linear to branched micelles [30,40], is not large. So although it could be used also for the present systems as a descriptor to differentiate between branched and linear micelles, we want to show in the following how other non-linear flows can give a more differentiated picture of this transition.

Conversely to the shear viscosity, no maximum is observed in the elastic modulus with increasing total surfactant concentration. The elastic modulus is observed to increase monotonically with increasing total surfactant concentration. Thus even as the viscosity passes through a plateau, the theoretical mesh size, $\zeta_{m}=\left(k_{B} T / G_{0}\right)^{1 / 3}$, is observed to monotonically decrease meaning that the proximity of entanglement points and the density of the wormlike micelle mesh continue to increase with increasing surfactant concentration $[55,56]$. Here $k_{B}$ is the Boltzmann constant and $T$ is the temperature. For wormlike micelles, theoretical predications and experiments have demonstrated that the plateau modulus for an entangled system should increase as $G_{0} \propto c^{9 / 4}$ with increasing concentration [37, 44]. Finally, we see an increase in the breakup time with decreasing total surfactant concentration in good agreement with the literature [44].

\subsection{Large amplitude oscillatory shear rheology (LAOS)}

The large amplitude oscillatory shear behavior of the four wormlike micelle solutions tested is presented in Figure 4 for an angular frequency of $\omega=3 \mathrm{rad} / \mathrm{s}$ and increasing percent strain. The storage, $G_{1}^{\prime}$, and loss modulus, $G_{1}^{\prime \prime}$, were found to be independent of percent strain until about twenty or thirty percent, indicating linear viscoelastic behavior below that point. The storage moduli is approximately an order of magnitude larger than the loss modulus for all the fluids tested because the angular frequency was chosen such that it was beyond the cross over point in Figure 3 for each case. Beyond the linear viscoelastic (LVE) limit, except for the case of the $6 \mathrm{wt} \% 85 / 15 \mathrm{NaOA} / \mathrm{C}_{8} \mathrm{TAB}$, the elastic moduli were all observed to decline with increasing strain, whereas the loss moduli for most cases exhibited a weak overshoot before declining at large strains. Such plastic nonlinear behavior at large amplitudes is typical of weak gels [57] even those that are shear-thinning.

In the nonlinear viscoelastic region, higher harmonic stresses arise [7]. Although, the dominant response was found to be from the first harmonic, additional insights can be gained from the response due to higher harmonics contribution. The nonlinear response of the wormlike micelle solutions can be 
visualized graphically from the elastic Lissajous-Bowditch plots. These plots of stress versus strain are presented in Figure 5 for a series of linear and branched wormlike micelles solutions. As expected, for both the linear and branched wormlike micelle solutions, in the linear viscoelastic limit at small strains, the resulting elastic Lissajous-Bowditch plots were found to be straight lines. In the nonlinear regime, the plots become distorted due to the contribution of higher order harmonics. It is in the large strain regime that the differences between the linear and branched wormlike micelles become apparent. For the linear wormlike micelles, both the $3 \mathrm{wt} \% 70 / 30 \mathrm{NaOA} / \mathrm{C}_{8} \mathrm{TAB}$ and the $6 \mathrm{wt} \% 85 / 15 \mathrm{NaOA} / \mathrm{C}_{8} \mathrm{TAB}$ solutions, the nonlinear response at large strains presents itself as modest distortion in the Lissajous-Bowditch plots resulting from the growth of the third harmonic and an evolution from a primarily elastic to a more viscous response. Even at strains approaching $1000 \%$ the distortion from the elliptical shape of the LVE response was not found to be too dramatic, although we should note that the Lissajous-Bowditch plots of the $6 \mathrm{wt} \% 85 / 15 \mathrm{NaOA} / \mathrm{C}_{8} \mathrm{TAB}$ case showed more distortion than the $3 \mathrm{wt} \% 70 / 30 \mathrm{NaOA} / \mathrm{C}_{8} \mathrm{TAB}$ solution. A very different response was observed for the branched wormlike micelle systems. Here, even at quite modest strains of $50 \%$ to $100 \%$ significant departures from the LVE response were observed for both branched systems with the growth of a strong third and then fifth harmonic in the data. With a larger surfactant concentration and similarly a larger likelihood of micelle branching, the deviation from LVE behavior was found to become more pronounced and onset of significant deviations were observed to occur at smaller applied strains. At large strains, a strong strain softening is observed in the LissajousBowditch plot. As the applied strain was increased, these Lissajous-Bowditch plots became more distorted eventually resulting in a negative elastic moduli for strains above $400 \%$ and $100 \%$ for $4.5 \mathrm{wt} \%$ $70 / 30 \mathrm{NaOA} / \mathrm{C}_{8} \mathrm{TAB}$ and $6 \mathrm{wt} \% 70 / 30 \mathrm{NaOA} / \mathrm{C}_{8} \mathrm{TAB}$ respectively. The negative elastic moduli has been shown to be related to the stress overshoot that is often observed in the startup of steady shear flows [2, 58]. This stress overshoot is a characteristic of materials that undergo significant microstructural changes [15]. Ewoldt et al. [2] demonstrated that wormlike micelle solutions, which can break down and reform under flow, can exhibit a negative elastic modulus in LAOS. For the systems being studied here, it appears that one fingerprint of branching might be the appearance of a negative elastic modulus which likely results from the additional relaxation mechanisms (ghost-like crossings and sliding branch points) which are available to the branched wormlike micelle solutions, but not the linear wormlike micelle solutions.

The nonlinearities of the wormlike micelle solutions were further examined through the local viscoelastic material properties quantified within an oscillatory cycle. The minimum-strain dynamic moduli, $G_{M}^{\prime}$, and large-strain dynamic moduli, $G_{L}^{\prime}$, are shown in Figure 6 as a function of strain amplitude for an applied angular frequency of $3 \mathrm{rad} / \mathrm{s}$. In the LVE region, these measures reduce to the 
first-harmonic storage modulus, $G_{1}^{\prime}$, as expected. In the nonlinear region, for all the wormlike micelle solutions tested, both $G_{M}^{\prime}$ and $G_{L}^{\prime}$ were found to decrease with increasing strain amplitude which indicates a softening behavior with increasing imposed strain between oscillatory cycles. The second apparent difference between the linear and branched wormlike micelles in LAOS can be observed in the rate of change of the moduli in Figures 4 and 6 . Here we observe a decay rate of nearly $G_{b r a n c h e d}^{\prime} \propto \gamma^{-2}$ for the branched wormlike micelles until an applied strain of, beyond which, the elastic modulus of the branched micelle systems becomes negative. Conversely, the linear wormlike micelles decay at a much slower rate, roughly as $G_{\text {linear }}^{\prime} \propto \gamma^{-1}$. So it is clear that the branched wormlike micelles are much more sensitive to the amplitude of the applied strain. Additionally, with increasing strain amplitude, the minimum-strain (maximum-strain rate) modulus, $G_{M}^{\prime}$, of the branched wormlike micelles at medium strain amplitudes was found to decrease more slowly than the large-strain (minimum-strain rate) modulus, $G_{L}^{\prime}$, in the range where the moduli were positive. For the linear wormlike micelles, the opposite trend was observed.

The elastic nonlinearities were further examined through the measure, stiffening ratio, $S$, plotted as a function of applied strain amplitude in Figure 7. In the linear viscoelastic limits, where the elastic Lissajous-Bowditch plots appear as straight lines, the stiffening ratio is exactly zero, $S=0$. As described in the theoretical background section, the stiffening ratio given by equation 10 describes whether the elastic modulus softens, $S<0$, or stiffens, $S>0$, with increasing strain within an oscillatory cycle. In the nonlinear region, beyond a strain of $20 \%$ for the most heavily branched wormlike micelle system, the LAOS behavior within an oscillatory cycle was found to be strain-softening, $S<0$, up to a strain amplitude of approximately $100 \%$. Beyond a strain amplitude of $\gamma_{0}>100 \%$, the stiffening ratio was found to become positive and grow to values greater than $S>10$. This dramatic increase in the stiffening ratio coincides with the observation of a negative minimum strain moduli, $G^{\prime}{ }_{M}<0$. Ewoldt and McKinley [58] showed that this negative minimum strain modulus is a direct result of the non-linear response of the fluid and the growth of the higher harmonics in the data (see Equation 5). They showed that once the magnitude of the third harmonic of the elastic component in the data becomes larger than one third the strength of the fundamental frequency,,$e_{3}>1 / 3 e_{1}$, a negative minimum strain modulus will be observed, $G_{M}^{\prime}<0$ [58]. The response of the linear systems is again quite different. In the nonlinear region, beyond a strain of $200 \%$ for the linear wormlike micelle system, the LAOS behavior within an oscillatory cycle was found to be only strain-stiffening, $S>0$, until the maximum strain amplitude imposed. This can be observed in the elastic Lissajous-Bowditch plot in Figure 5 as a modest upturn in the stress at large strains. However, the behavior is in contrast to the large-strain dynamic moduli, $G_{L}^{\prime}$, which was found to 
soften with increasing imposed strain amplitude as seen in Figure 6. This discrepancy is the result of the definition of the stiffening ratio and can lead to misinterpretation of the results. The stiffening ratio can be greater than zero, $S>0$, if the large strain modulus, $G_{L}^{\prime}$, stiffens more quickly than the minimum strain modulus, $G_{M}^{\prime}$, or if $G_{M}^{\prime}$ softens more quickly than $G_{L}^{\prime}$. Thus $S>0$ can be interpreted as either strainhardening or strain-rate softening of the material and only through examination of $G_{L}^{\prime}$ and $G_{M}^{\prime}$ can a distinction be made and the data properly interpreted. For the case of the linear micellar systems in Figure 5, 6 and 7 this means that the intracycle strain-hardening $S$ increases with strain, but the overall response of $G_{L}^{\prime}$ and $G_{M}^{\prime}$ is a softening of the system with increasing strain and rate.

In an oscillatory cycle, the strain and strain-rate vary orthogonally. As a result, within an oscillatory cycle, the strain rate goes to zero as the strain is maximized and vice versa. The elastic stress response within a given cycle can thus be affected by either the varying strain and/or the varying strainrate. For the case of these linear wormlike micelle solutions, based on the measurements of $G_{M}^{\prime}$ and $G_{L}^{\prime}$ the dominant nonlinearity within the cycle is a softening with increasing strain-rate. While for the nonlinearity for the wormlike micelles is predominantly strain softening until 100\% strain for $6 \mathrm{wt} \% 70 / 30$ $\mathrm{NaOA} / \mathrm{C}_{8} \mathrm{TAB}$ and $400 \%$ strain for $4.5 \mathrm{wt} \% 70 / 30 \mathrm{NaOA} / \mathrm{C}_{8} \mathrm{TAB}$ case as indicated by the negative stiffening ratio. Beyond these strain amplitudes, a clear physical interpretation based on the stiffening ratio could not be made due to the complex sigmoidal-shaped elastic Lissajous-Bowditch plots. These complex shapes are associated with a strong elastic non-linearity [58].

Similarly, the corresponding viscous nonlinearities can be examined graphically from viscous Lissajous-Bowditch plots. The stress versus strain-rate plots of the wormlike micelle solutions tested are presented in Figure 8. For the linear wormlike micelles, the nonlinear response at large strain rates presents itself as a very slight distortion in the Lissajous-Bowditch plots resulting from the growth of the third harmonic. As seen in Figure 8, the viscous component of the stress is essentially linear even at the largest strains tested. The result as we will demonstrate explicitly in Figure 10 is little to no thinning or thickening of the viscosity within a cycle. A very different response was observed for the branched wormlike micelle systems. Here, even at quite modest strains of $50 \%$ to $100 \%$ a significant departure from the LVE response was observed with the growth of a strong third and then fifth harmonic in the data. At large strains a strong thickening of the dynamic viscosity was observed within a cycle. As the applied strain was increased, these Lissajous-Bowditch plots became more distorted eventually resulting in a secondary loop and a crossover in the measured stress as a function of strain rate for applied strains above $100 \%$ and $400 \%$ for $6 \mathrm{wt} \% 70 / 30 \mathrm{NaOA} / \mathrm{C}_{8} \mathrm{TAB}$ and $4.5 \mathrm{wt} \% 70 / 30 \mathrm{NaOA} / \mathrm{C}_{8} \mathrm{TAB}$ respectively. These loops occur with the onset of the negative elastic moduli seen in Figures 5 and 6 and are the result 
of a stress overshoot. A detailed discussion about these secondary loops and negative moduli including predictions of these phenomena using a Giesekus model can be found in Ewoldt et al. [58]. The size and strength of the observed secondary loops was found to increase with increasing applied strain.

The viscous nonlinearity quantified through the measures, minimum strain-rate dynamic viscosity, $\eta_{M}^{\prime}$, and the large strain-rate dynamic viscosity, $\eta_{L}^{\prime}$, are shown in Figure 9 . Both the measures showed initial mild thickening behavior at medium strain amplitudes and strong thinning behavior at large strain amplitudes. For the branched wormlike micelles, the local dynamic viscosity measures are not presented in Figure 9 for strain amplitudes at which secondary loops in the viscous LissajousBowditch plots are present due to the lack of clear physical interpretation of those measurements. However, at least until the onset of the secondary loops, the nonlinearity of the large strain rate dynamic viscosity, $\eta_{L}^{\prime}$, was found to be stronger than the minimum strain rate dynamic viscosity, $\eta_{M}^{\prime}$. This suggests a thickening of the fluid viscosity with increasing strain rates. The viscous nonlinearities are quantified using the shear-thickening ratio, $T$, and are presented in Figure 10. As described in the theoretical background section, the shear- thickening ratio given by equation 11 describes whether the viscosity thins, $T<0$, or thickens, $T>0$, with increasing strain rate within a cycle. The branched wormlike micelle solutions were found to thicken within a cycle much more significantly than the linear wormlike micelles resulting in a maximum thickening ratio, $T$, which was nearly three times as large.

\subsection{Orthogonal Superposition Rheology}

Orthogonal superposition rheology measurements were performed for the four wormlike micelle solutions presented in the previous section. In Figures 11-14, the orthogonal storage and loss moduli, $G_{\perp}^{\prime}$ and $G_{\perp}^{\prime \prime}$ for each of the wormlike micelle solutions for several shear rates up to $\gamma=3 \mathrm{~s}^{-1}$ and an oscillatory strain of 5\% are presented. The orthogonal moduli were verified against the rotational SAOS measurements (Figure 3) and were found to be consistent within roughly 10-20\% across the entire frequency range. The differences observed were likely the result of a pumping flow that occurs during the vertical oscillations of the Couette fixture. To minimize the effect of the pumping flow on the rheological measurements, $5 \mathrm{~mm}$ tall slits were machined into the inner wall of the Couette cell to allow fluid to be pumped into and out of the reservoir at the center of the Couette cell as seen in Figure 1 [5].

Starting with Figure 11 and moving through Figure 13, the total surfactant concentration is increased from $3 \%$ to $6 \%$ while keeping the relative concentration of the co-surfactants constant at 70/30 $\mathrm{NaOA} / \mathrm{C}_{8} \mathrm{TAB}$. With increasing concentration, the wormlike micelle solutions go from linear to branched. As can be seen from these figures, a number of similarities and clear differences can be 
observed as one moves from linear to branched systems. In each case, both the storage and loss moduli decrease at low frequencies with increasing applied shear rate. The result is a clear reduction in the dynamic viscosity measured from the orthogonal loss modulus as well as a reduction in the relaxation time. These results are consistent with previous orthogonal superposition measurements of wormlike micelles in the literature [4].

In all cases, deviations of the moduli from the regular SAOS results (with no applied shear) begin when the Weissenberg number is greater than one, $W i=\lambda \gamma>1$. Comparing the response of the linear wormlike micelles to the branched wormlike micelles, it appears that the presence of branches makes the SAOS more sensitive to the orthogonal applied steady shear. This can be seen more explicitly in Figure 15 where the plateau modulus and viscosity obtained by fitting the data in Figures 11-14 to a Maxwell model are presented as a function of the applied orthogonal shear rate. If one focuses initially on the viscosity dependence, we observe a much larger change for the branched wormlike micelles with applied shear rate as compared to the linear systems. If one fits the viscosity data with a power law, one finds a slope of $\eta \propto \gamma^{-0.35}$ for the linear wormlike micelles (3wt $\left.\% 70 / 30 \mathrm{NaOA} / \mathrm{C}_{8} \mathrm{TAB}\right)$ and a slope of nearly twice that $\eta \propto \gamma^{-0.68}$ for the branched micelles $\left(6 \mathrm{wt} \% 70 / 30 \mathrm{NaOA} / \mathrm{C}_{8} \mathrm{TAB}\right)$ with the $4.5 \mathrm{wt} \% 70 / 30$ $\mathrm{NaOA} / \mathrm{C}_{8} \mathrm{TAB}$ in between these results. Note, that the decay with increasing shear rate is expected as the underlying entangled micelle network is being stretched and broken by the applied shear. Interestingly, however, if one inspects the steady shear data in Figure 4, one observes that no clear distinction can be observed between linear and branched systems. They decay with a quite similar power law exponent.

At large frequencies, the effect of orthogonal shear on the plateau modulus can be systematically studied. Here we see clear differences between the branched and the linear systems. The plateau modulus of the linear systems in Figures 11 and 14 were found to be insensitive to applied shear rate. This is similar to observations made for polymer solutions [6], but opposed to the measurements made for wormlike micelle solutions in the past [4]. This observation suggests that for linear wormlike micelles the orthogonal shear is deforming the entangled micelle network. If the micelles were in fact being broken by the applied shear one would expect a change in the plateau modulus because the plateau modulus can be directly related to the mesh size of the entangled micelle network, $\zeta_{m}=\left(k_{B} T / G_{0}\right)^{1 / 3}$. A breakdown of the micelles and a growth in the mesh size is, however, observed for the branched wormlike micelle solution. In Figure 13, we can see a significant reduction in the plateau modulus with imposed shear rate. The changes of the plateau modulus with shear rate are explicitly presented in Figure 15. Note that in order to obtain the data in Figure 15, the data from Figures 11-14 were first fit with a single mode Maxwell model in order to extract the viscosity, relaxation time and plateau modulus. For the 
branched micelles, a change from a plateau modulus of $G=100 \mathrm{~Pa}$ with zero applied shear to $G=70 \mathrm{~Pa}$ at a shear rate of $\gamma=1 \mathrm{~s}^{-1}$ was observed. The result is a change in mesh size from $34 \mathrm{~nm}$ to $39 \mathrm{~nm}$ which is an increase of $15 \%$.

Finally, we inspect the minimum in the $G_{\perp}^{\prime \prime}$, which we know can be correlated with the break-up time of the micelles, $\lambda_{b r} \approx 1 / \omega_{\min }$. Here we note that because of the noise in the data, the exact value of the breakup time as a function of shear rate cannot be precisely determined, however, we should note that no obvious shift in the breakup time is observed for either the branched or the linear wormlike micelle solutions even as the relaxation time derived from fits to the Maxwell model was found to vary by almost two orders of magnitude in some cases. Let's take for example the case of the branched wormlike micelle solution. The relaxation time at zero shear rate is $\lambda=790 \mathrm{~s}$ and decays to $\lambda=26 \mathrm{~s}$ at a shear rate of

$\gamma=1 \mathrm{~s}^{-1}$. Given that $\lambda=\left(\lambda_{b r} \lambda_{\text {rep }}\right)^{1 / 2}$, this suggests that the reptation time has decayed by a factor of more than 1000 over that same range in orthogonal shear rates. Over the same range of shear rates the reptation time of the linear wormlike micelle solution $\left(3 \mathrm{wt} \% 70 / 30 \mathrm{NaOA} / \mathrm{C}_{8} \mathrm{TAB}\right)$ was only found to decay by a factor of just 28 .

\section{Conclusions}

In this study two non-linear rheology tools, orthogonal superposition rheology and large amplitude oscillatory shear rheology, were used to investigate whether a non-linear rheological signature could be found which demonstrates the transition from a linear to a branched wormlike micelle solution. A series of mixtures of an anionic and a cationic surfactant known to produce high viscosity wormlike micelle solutions were used. The surfactants were octyl trimethyl ammonium bromide $\left(\mathrm{C}_{8} \mathrm{TAB}\right)$ and sodium oleate $(\mathrm{NaOA})$. A series of linear and branched wormlike micelle solutions were obtained by varying the relative ratio of $\mathrm{NaOA}$ to $\mathrm{C}_{8} \mathrm{TAB}$ at a fixed total surfactant concentration or by fixing the ratio of $\mathrm{NaOA}$ to $\mathrm{C}_{8} \mathrm{TAB}$ and varying the total surfactant concentration.

Orthogonal superposition measurements were conducted with varying rotational shear rates in the range of $0<\gamma_{r \theta}<10 \mathrm{~s}^{-1}$ and superimposing a small amplitude oscillatory shear flow with angular frequencies varying from $0.001<\omega<100 \mathrm{rad} / \mathrm{s}$ orthogonal to the rotational shear. The plateau modulus and the relaxation times of linear and branched cases were probed as a function of the imposed steady orthogonal shear rates. The plateau modulus and the relaxation-times of the branched wormlike micelle solutions were both found to be more sensitive to the strength of the imposed orthogonal rotational shear flow than the linear wormlike micelle solutions, both decreasing with increasing shear rate. This is likely due to the additional stress relaxation mechanism available to branched wormlike micelle solutions under 
flow, namely sliding branch points and ghost-like crossings, and to the break-down of the branched structure which can be directly inferred from the sharp decrease observed in the plateau modulus which, from rubber elasticity theory, is known to be inversely proportional to the cube of the mesh size of the entangled micelle network. These relaxation mechanisms were also seen in the more than 1000x decrease in the reptation time for the branched wormlike micelles at the largest imposed shear rate. At those same rates a factor of only 20x was observed for the linear wormlike micelles.

In the Large Amplitude Oscillatory Shear (LAOS) presented in this paper the imposed oscillations angular frequency was fixed at $\omega=3 \mathrm{rad} / \mathrm{s}$ and the strain amplitude was increased for up to $\gamma_{0}=1000 \%$. In these measurements, both the linear and branched wormlike micelle solutions exhibited a qualitatively similar trend in the viscoelastic non-linearities with increasing strain amplitude. However, the strength of the viscoelastic non-linearities of the branched wormlike micelle solutions, both within an oscillatory cycle and with increasing strain amplitude, were found to be significantly larger than the viscoelastic non-linearities observed for the linear wormlike micelle solutions. An obvious difference between the nonlinear response of the branched and linear wormlike micelle solutions was found in the Lissajous-Bowditch plots. As the applied strain was increased, the Lissajous-Bowditch plots of the linear wormlike micelles solutions were not greatly affected, however, the Lissajous-Bowditch plots of the branched wormlike micelle solutions became much more distorted eventually resulting in a secondary loop and a crossover in the measured stress as a function of strain rate for applied strains above $100 \%$. These loops occur with the onset of a negative elastic moduli and are the result of stress overshoot that can be observed in the start-up of shear flow. The presence of the stress overshoot and the negative elastic moduli is likely the result of the breakdown of the underlying wormlike micelle entangled structure. A failure, that is made more likely due to the added stress relief mechanisms, namely ghost-like crossings and sliding branch points, available to branched wormlike micelles solutions, but not linear wormlike micelle solutions. Thus these LAOS observations are consistent with the physical insights gained into the evolution of the microstructure of both linear and wormlike micelles from the orthogonal superposition measurements.

As both orthogonal superposition rheology and LAOS probe the non-linear behavior of the wormlike micelle solutions, a direct, one-to-one, comparison of the results outside of the linear regime cannot be made. We will, however, use this space to present what we feel is an unbiased comparison of the pros and cons of these two non-linear rheology techniques. The major positive feature of orthogonal superposition rheology is also the major negative feature of LAOS; ease of interpretation. Orthogonal superposition rheology uses the same standard rheological material properties (storage/loss modulus, plateau modulus, dynamic viscosity and relaxation time) that rheologists are already familiar with and for 
which they have already acquired an intuitive feel. As a result, changes to the fluid microstructure can be directly inferred from variations in relaxation time and plateau modulus. For instance, in the case of the branched wormlike micelle solutions studied here, the variation in the plateau modulus with imposed shear could be directly correlated to changes in the entangled network's mesh size while the variation in the location of the minimum in the loss modulus could be correlated to variations in the micelle breakup time. LAOS on the other hand is much more difficult to physically interpret in part because it requires the use of a host of new non-linear viscoelastic material properties. However, because of the wealth of new material properties and their sensitivity to experimental conditions, LAOS is an excellent tool for making both qualitative and quantitative comparisons between different fluids. In LAOS, as in orthogonal superposition rheology, physical interpretation of the results can be aided by fitting the predictions of different constitutive models to the data.

As described in detail above, orthogonal superposition rheology and LAOS are quite different non-linear measurement techniques. Orthogonal superposition measures the linear response of a fluid that has been deformed by the imposition of an orthogonal shear flow that has reached steady state. As a result, as currently implemented, only steady state information can be retrieved by orthogonal superposition measurements. Conversely, LAOS can directly probe the transient evolution of the nonlinear viscoelastic material properties over a very wide strain and strain-rate space. However, within a LAOS oscillatory cycle, the strain and strain rate vary orthogonally. As a result, as the strain is maximized within a cycle, the strain rate goes to zero and vice versa. Thus the nonlinear viscoelastic response within a given cycle can be affected by the varying strain and/or the varying strain-rate. Deconvoluting the effects of each on the fluid's response can be challenging, but will give insight into whether the observed non-linearities are the result of sensitivity to changes in strain or strain rate. Finally, it should be noted that typically the LAOS response is only analyzed after tens or hundreds of oscillation cycles. Thus, in LAOS a steady state response is also achieved and as a result only evolution in the fluid structure that can be built up and broken down over an LAOS oscillation cycle can be probed.

Finally, we discuss the implementation and availability of each technique. Here LAOS has a clear advantage as it is commercially available on most major rheometers with analysis software included or available for free download (MITlaos). On the other hand, until the recent release of the TA instruments fixture, orthogonal superposition was only available through custom built systems. Orthogonal superposition is limited by the sensitivity of the normal force transducer, the quality of the instrument calibration and the pumping flow that results from the axial oscillations to working with fluids with moderate to high viscosities. LAOS is also limited by the sensitivity of the torque transducer and by the inertial effects for controlled stress rheometer to moderate to high viscosity fluids and moderate to low 
frequencies. Finally, it should be pointed out that the post processing of LAOS data can be quite time consuming and the quality of the data is often masked by the rheometer software. As a result, double checking of the raw data for each experiment is critical.

In conclusion, we believe both LAOS and orthogonal superposition rheology are excellent tools for probing the non-linear response of a viscoelastic fluid. Each has its strengths and weaknesses. Which technique one choses must therefore depend on one's rheological needs and the availability of the experimental instrumentation.

\section{Acknowledgements}

The authors would like to thank the National Science Foundation for their generous support of SK and JPR under the UMASS NSEC Center for Hierarchical Manufacturing NSF Grant CMMI-1025020 as well as the Research Council of KU Leuven for the support of JPR under the fellowship number SF/14/018, and the People Programme (Marie Curie Actions) of the European Union's Seventh Framework Programme (FP7/2007-2013) for support of JH and CJHC under REA grant agreement number 607937 (SUPOLEN).

\section{References}

[1] Hyun, K., M. Wilhelm, C. O. Klein, K. S. Cho, J. G. Nam, K. H. Ahn, S. J. Lee, R. H. Ewoldt, and G. H. McKinley, "A review of nonlinear oscillatory shear tests: Analysis and application of large amplitude oscillatory shear (laos),' Progress in Polymer Science 36, 1697 1753 (2011).

[2] Ewoldt, R. H., A. E. Hosoi, and G. H. McKinley, "New measures for characterizing nonlinear viscoelasticity in large amplitude oscillatory shear,' J. Rheol. 52, 1427-1458 (2008).

[3] Wilhelm, M., 'Fourier-transform rheology,'” Macromol. Mat. \& Eng. 287, 83-105 (2002).

[4] Kim, S., J. Mewis, C. Clasen, and J. Vermant, "Superposition rheometry of a wormlike micellar fluid," Rheol. Acta 52, 727-740 (2013).

[5] Vermant, J., P. Moldenaers, J. Mewis, M. Ellis, and R. Garritano, “'Orthogonal superposition measurements using a rheometer equipped with a force rebalanced transducer,' Rev. Sci. Instrum. 68, 4090-4096 (1997).

[6] Vermant, J., L. M. Walker, P. Moldenaers, and J. Mewis, “Orthogonal versus parallel superposition measurements,'” J. Non-Newtonian Fluid Mech. 79, 173-189 (1998).

[7] Wilhelm, M., D. Marin, and H. W. Spiess, "Fourier-transform rheology," Rheol. Acta 37, 399-405 (1998).

[8] Wilhelm, M., P. Reinheimer, and M. Ortseifer, "High sensitivity fourier-transform rheology,', Rheol. Acta 38, 349-356 (1999).

[9] Leblanc, J. L., M. Putman, and E. Pianhanuruk, "A thorough study on the relationships between dispersion quality and viscoelastic properties in carbon black filled sbr compounds,' J. App. Polym. Sci. 121, 1096-1117 (2011).

[10] Leblanc, J. L., "Large amplitude oscillatory shear experiments to investigate the nonlinear viscoelastic properties of highly loaded carbon black rubber compounds without curatives,' J. 
App. Polym. Sci., 1271-1293 (2008).

[11] Hyun, K., G. J. Nam, M. Wilhelm, K. H. Ahn, and S. J. Lee, "Nonlinear response of complex fluids under laos (large amplitude oscillatory shear) flow,' Korea-Australia Rheol. J. 15, 97-105 (2003).

[12] Hyun, K., J. G. Nam, M. Wilhelm, K. H. Ahn, and S. J. Lee, 'Large amplitude oscillatory shear behavior of peo-ppo-peo triblock copolymer solutions,', Rheol. Acta 45, 239-249 (2006).

[13] Neidhfer, T., S. Sioula, N. Hadjichristidis, and M. Wilhelm, "Distinguishing linear from star-branched polystyrene solutions with fourier-transform rheology,' Macromol. Rapid Commun. 25, 1921-1926 (2004).

[14] Klein, C., P. Venema, L. Sagis, and E. J. N.-N. F. M. Linden, "Rheological discrimination and characterization of carrageenans and starches by fourier transform-rheology in the non-linear viscous regime,' J. Non-Newtonian Fluid Mech. 151, 145-150 (2008).

[15] Bird, R. B., R. C. Armstrong, and O. Hassager, Dynamics of polymeric liquids: Volume 1 fluid mechanics (John Wiley \& Sons, New York, 1987).

[16] Atalik, K., and R. Keunings, "On the occurrence of even harmonics in the shear stress response of viscoelastic fluids in large amplitude oscillatory shear,' J. Non-Newtonian Fluid Mech. 122, 107-116 (2004).

[17] Graham, M. D., " Wall slip and the nonlinear oscillatory dynamics of large amplitude shear flows,' J. Rheol. 39, 697-712 (1995).

[18] Cho, K. S., K. Hyun, K. H. Ahn, and S. J. Lee, " A geometrical interpretation of large amplitude oscillatory shear response,' J. Rheol. 49, 747-758 (2005).

[19] Israelachvili, J. N., Intermolecular and surface forces: With applications to colloidal and biological systems (Academic Press, London, 1985).

[20] Larson, R. G., The structure and rheology of complex fluids (Oxford University Press, New York, 1999).

[21] Rehage, H., and H. Hoffmann, "Viscoelastic surfactant solutions: Model systems for rheological research," Mol. Phys. 74, 933-973 (1991).

[22] Zana, R., and E. Kaler, "Giant micelles, properties and applications," in Editor (ed)^(eds). Book Giant micelles, properties and applications (CRC Press, 2007).

[23] Cates, M. E., "Reptation of living polymers: Dynamics of entangled polymers in the presence of reversible chain-scission reactions,' Macromol. 20, 2289-2296 (1987).

[24] Olmsted, P. D., "Dynamics and flow induced phase separation in polymeric fluids," Current Opinion in Colloid and Interface Science 4, 95-100 (1999).

[25] Shikata, T., and T. Kotaka, "Entanglement network of thread-like micelles of a cationic detergent,' J. Non-Crystal. Sol. 131-133, 831-835 (1991).

[26] Rothstein, J. P., "Strong flows of viscoelastic wormlike micelle solutions," in Binding, D. M. and K. Walters (eds). Rheology reviews (The British Society of Rheology, Aberystwyth, Wales, UK, 2008).

[27] Shikata, T., H. Hirata, and T. Kotaka, "Micelle formation of detergent molecules in aqueous-media - viscoelastic properties of aqueous cetyltrimethylammonium bromide solutions,', Langmuir 3, 1081-1086 (1987).

[28] Lequeux, F., and S. J. Candau, "Structural properties of wormlike micelles," in McLeish, T. (ed). Theoretical challenges in the dynamics of complex fluids (Kluwer Academic Publishers, Netherlands, 1997).

[29] Danino, D., A. Bernheim-Groswasser, and Y. Talmon, "Digital cryogenic transmission electron microscopy: An advanced tool for direct imaging of complex fluids,' Colloids and 
Surfaces A: Physicochemical and Engineering Aspects 183, 113-122 (2001).

[30] Cui, H., T. K. Hodgdon, E. W. Kaler, L. Abezgauz, D. Danino, M. Lubovsky, Y. Talmon, and D. J. Pochan, "Elucidating the assembled structure of amphiphiles in solution via cryogenic transmission electron microscopy,', Soft Matter 3, 945 - 955 (2007).

[31] Angelico, R., S. Amin, M. Monduzzi, S. Murgia, U. Olssone, and G. Palazzo, “Impact of branching on the viscoelasticity of wormlike reverse micelles,' Soft Matter 9, 10941 (2012).

[32] Palazzo, G., “'Wormlike reverse micelles,' Soft Matter 9, 10668 (2013).

[33] Dhakal, S., and R. Sureshkumar, "Topology, length scales, and energetics of surfactant micelles,' J. Chem. Phys. 143, 024905 (2015).

[34] Hassan, P. A., S. J. Candau, F. Kern, and C. Manohar, "Rheology of wormlike micelles with varying hydrophobicity of the counterion,'” Langmuir 14, 6025-6029 (1998).

[35] Khatory, A., F. Lequeux, F. Kern, and S. J. Candau, "Linear and nonlinear viscoelasticity of semidilute solutions of wormlike micelles at high salt concentration,' Langmuir 9, 1456-1464 (1993).

[36] Appell, J., G. Porte, A. Khatory, F. Kern, and S. J. Candau, "Static and dynamic properties of a network of wormlike surfactant micelles (etylpyridinium chlorate in sodium chlorate brine),' J. Phys. II 2, 1045-1052 (1992).

[37] Koehler, R. D., S. R. Raghavan, and E. W. Kaler, "Microstructure and dynamics of wormlike micellar solutions formed by mixing cationic and anionic surfactants,' J. Phys. Chem. B 104, 11035-11044 (2000).

[38] Raghavan, S. R., G. Fritz, and E. W. Kaler, "Wormlike micelles formed by synergistic selfassembly in mixtures of anionic and cationic surfactants,', Langmuir 18, 3797-3803 (2002).

[39] Angelescu, D., A. Khan, and H. Caldararu, "Viscoelastic properties of sodium dodecyl sulfate with aluminum salt in aqueous solution,' Langmuir 19, 9155-9161 (2003).

[40] Ziserman, L., "Relationship between rheological properties and nanostructure in mixed micellar surfactant," M.S., Technion Israel Institute of Technology, (2005).

[41] Drye, T. J., and M. E. Cates, "Living netwoks: The role of cross-links in entangled surfactant solutions,', J. Chem. Phys. 96, 1367-1375 (1992).

[42] Wagner, M. H., H. Bastian, P. Hachmann, J. Meissner, K. S., H. Munstedt, and F. Langouche, "The strain-hardening behavior of linear and long-chain-branched polyolefin melts in extensional flows,' Rheol. Acta 39, 97-109 (2000).

[43] Munstedt, H., and H. M. Laun, "Elongational properties and molecular structure of pollyethylene melts,', Rheol. Acta 20, 211-221 (1981).

[44] Chellamuthu, M., and J. P. Rothstein, "Distinguishing between linear and branched wormlike micelle solutions using extensional rheology measurements,' J. Rheol. 52, 865-884 (2008).

[45] Sachsenheimer, D., C. Oelschlaeger, S. Muller, J. Kustner, S. Bindgen, and N. Willenbacher, "Elongational deformation of wormlike micellar solutions,', J. Rheol. 58, 201742 (2014).

[46] Rothstein, J. P., “Transient extensional rheology of wormlike micelle solutions,' J. Rheol. 47, 1227-1247 (2003).

[47] Yesilata, B., C. Clasen, and G. H. McKinley, "Nonlinear shear and extensional flow dynamics of wormlike surfactant solutions,' J. Non-Newtonian Fluid Mech. 133, 73-90 (2006).

[48] Bhardwaj, A., E. Miller, and J. P. Rothstein, "Filament stretching and capillary breakup extensional rheometry measurements of viscoelastic wormlike micelle solutions,' J. Rheol. 51, 693-719 (2007). 
[49] Bhardwaj, A., D. Richter, M. Chellamuthu, and J. P. Rothstein, "The effect of preshear on the extensional rheology of wormlike micelle solutions," Rheol. Acta 46, 861-875 (2007).

[50] Merger, D., and M. Wilhelm, "Intrinsic nonlinearity from laostrain experiments on various strain- and stress-controlled rheometers: A quantitative comparison," Rheol. Acta 53, 621-634 (2014).

[51] Graessley, W. W., "Polymer chain dimensions and the dependence of viscoelastic properties on concentration, molecular weight and solvent power," Polymer 21, 258-262 (1980).

[52] Spenley, N. A., X. F. Yuan, and M. E. Cates, "Nonmonotonic constitutive laws and the formation of shear-banded flows," J. Phys. II France 6, 551-571 (1996).

[53] Fischer, P., and H. Rehage, "Rheological master curves of viscoelastic surfactant solutions by varying the solvent viscosity and temperature," Langmuir 13, 7012-7020 (1997).

[54] Kern, F., F. Lequeux, R. Zana, and S. J. Candau, "Dynamics properties of salt-free viscoelastic micellar solutions," Langmuir 10, 1714-1723 (1994).

[55] Doi, M., and S. F. Edwards, The theory of polymer dynamics (Oxford University Press, Oxford, 1986).

[56] Granek, R., and M. E. Cates, "Stress relaxation in living polymers: Results from a poisson renewal model," J. Chem. Phys. 96, 4758-4767 (1992).

[57] Sun, W. X., Y. R. Yang, T. Wang, X. X. Liu, C. Y. Wang, and Z. Tong, "Large amplitude oscillatory shear rheology for nonlinear viscoelasticity in hectorite suspensions containing poly(ethylene glycol),' Polymer 52, 1402-1409 (2011).

[58] Ewoldt, R. H., and G. H. McKinley, "On secondary loops in laos via self-intersection of lissajous-bowditch curves," Rheol. Acta 49, 213-219 (2010). 


\section{Figures and Tables}

\begin{tabular}{|c|c|c|c|c|}
\hline Sample Name & $G_{0}[\mathrm{~Pa}]$ & $\lambda[\mathrm{s}]$ & $\eta_{0}[\mathrm{~Pa}-\mathrm{s}]$ & $\lambda_{b r}[\mathrm{~s}]$ \\
\hline $3 \mathrm{wt} \% 70 / 30 \mathrm{NaOA} / \mathrm{C}_{8} \mathrm{TAB}$ & 8.5 & 20 & 170 & 0.83 \\
\hline $4.5 \mathrm{wt} \% 70 / 30 \mathrm{NaOA} / \mathrm{C}_{8} \mathrm{TAB}$ & 52 & 19 & 990 & 0.17 \\
\hline $6 \mathrm{wt} \% 70 / 30 \mathrm{NaOA} / \mathrm{C}_{8} \mathrm{TAB}$ & 100 & 7.8 & 791 & 0.063 \\
\hline $6 \mathrm{wt} \% 85 / 15 \mathrm{NaOA} / \mathrm{C}_{8} \mathrm{TAB}$ & 28 & 0.9 & 25 & - \\
\hline
\end{tabular}

Table 1: Maxwell model fits to the linear viscoelastic data of the four wormlike micelle solutions tested.

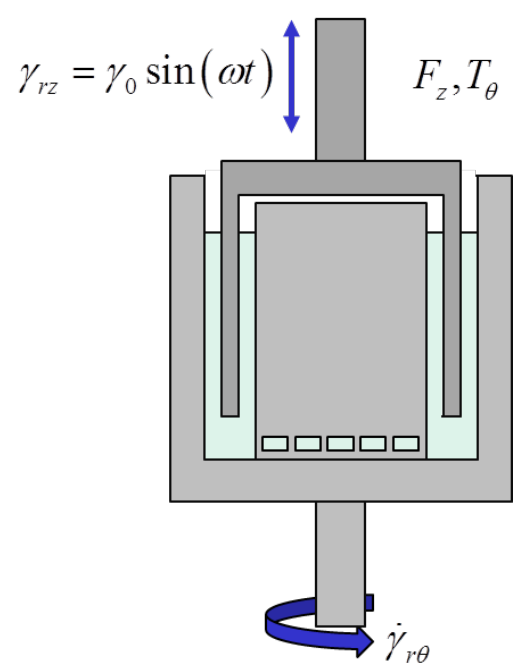

Figure 1: Schematic diagram of the orthogonal superposition setup using a specially-modified double wall Couette cell. 


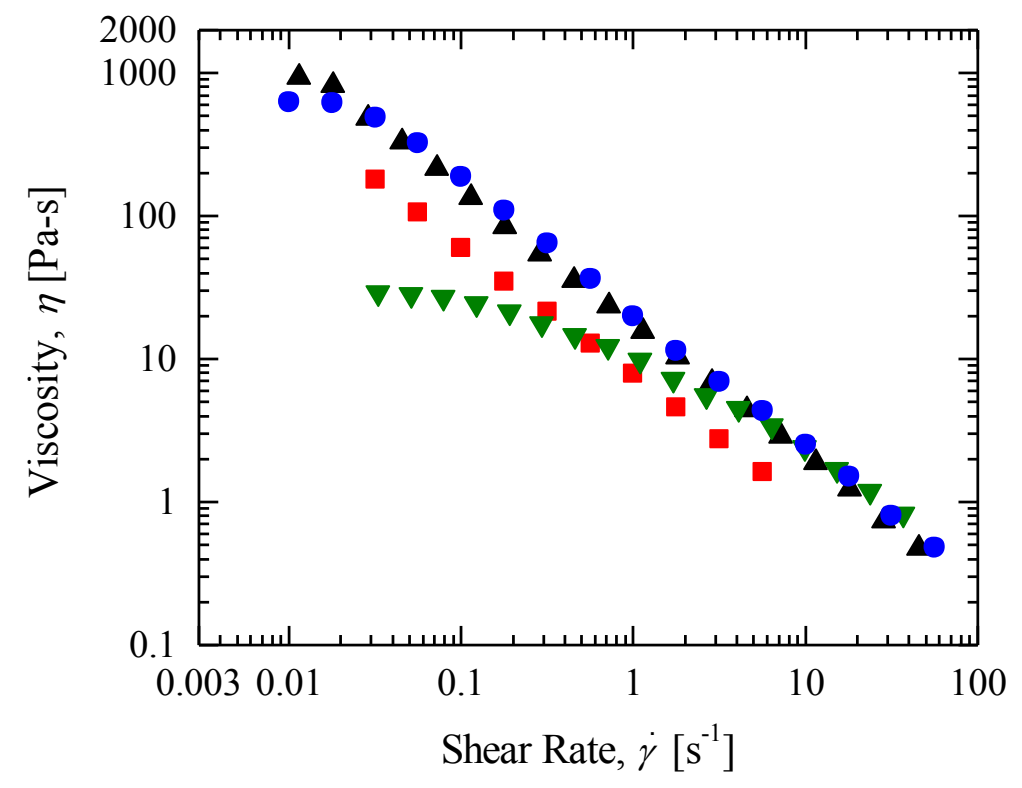

Figure 2: Steady shear viscosity as a function of shear rate for a series of wormlike micelle solutions

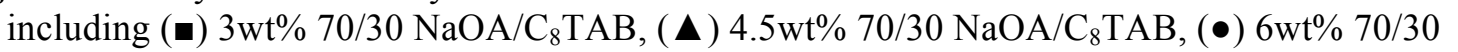
$\mathrm{NaOA} / \mathrm{C}_{8} \mathrm{TAB}$, and ( $) 6 \mathrm{wt} \% 85 / 15 \mathrm{NaOA} / \mathrm{C}_{8} \mathrm{TAB}$.

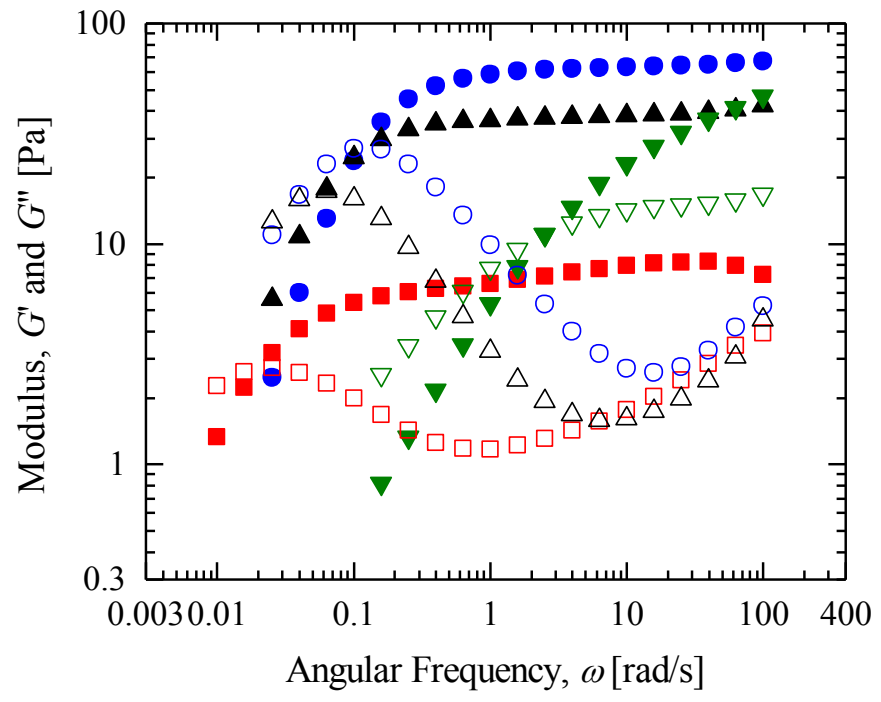

Figure 3: Small amplitude oscillatory shear measurements for a series of wormlike micelle solutions. Included in the figure are storage modulus (solid symbols) and loss modulus (hollow symbols) as a function of angular frequency for a series of wormlike micelle solutions including: (ש) $3 \mathrm{wt} \%$ 70/30 $\mathrm{NaOA} / \mathrm{C}_{8} \mathrm{TAB},(\boldsymbol{\Delta}) 4.5 \mathrm{wt} \% 70 / 30 \mathrm{NaOA} / \mathrm{C}_{8} \mathrm{TAB},(\bullet) 6 \mathrm{wt} \% 70 / 30 \mathrm{NaOA} / \mathrm{C}_{8} \mathrm{TAB}$, and (文) $6 \mathrm{wt} \% 85 / 15$ $\mathrm{NaOA} / \mathrm{C}_{8} \mathrm{TAB}$. 

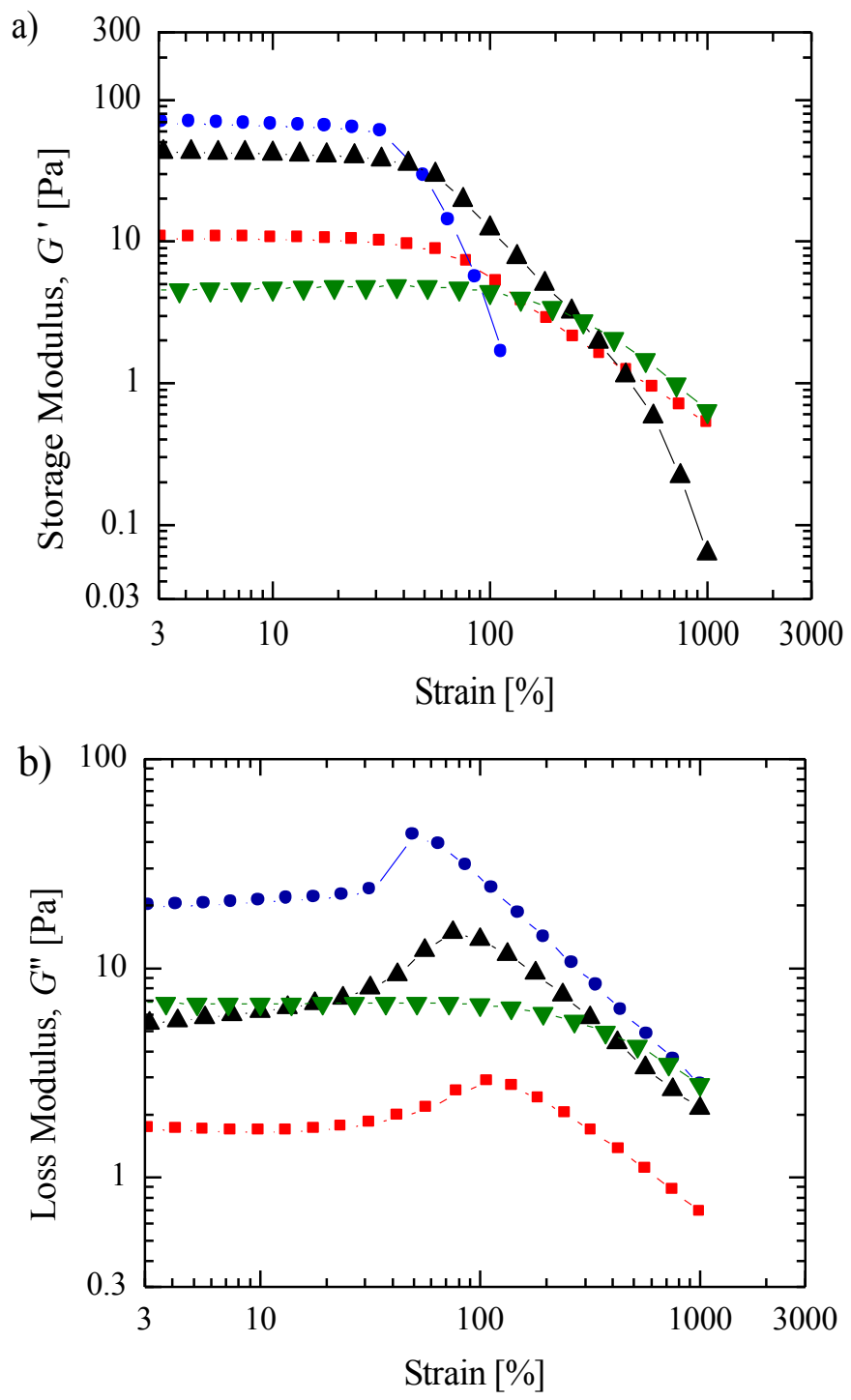

Figure 4: a) Storage modulus and b) loss modulus as a function of imposed strain for a series of wormlike micelle solutions including: (•) $3 \mathrm{wt} \% 70 / 30 \mathrm{NaOA} / \mathrm{C}_{8} \mathrm{TAB},(\boldsymbol{\Delta}) 4.5 \mathrm{wt} \% 70 / 30 \mathrm{NaOA} / \mathrm{C}_{8} \mathrm{TAB},(\bullet) 6 \mathrm{wt} \%$ $70 / 30 \mathrm{NaOA} / \mathrm{C}_{8} \mathrm{TAB}$, and $(\boldsymbol{\nabla}) 6 \mathrm{wt} \% 85 / 15 \mathrm{NaOA} / \mathrm{C}_{8} \mathrm{TAB}$. 

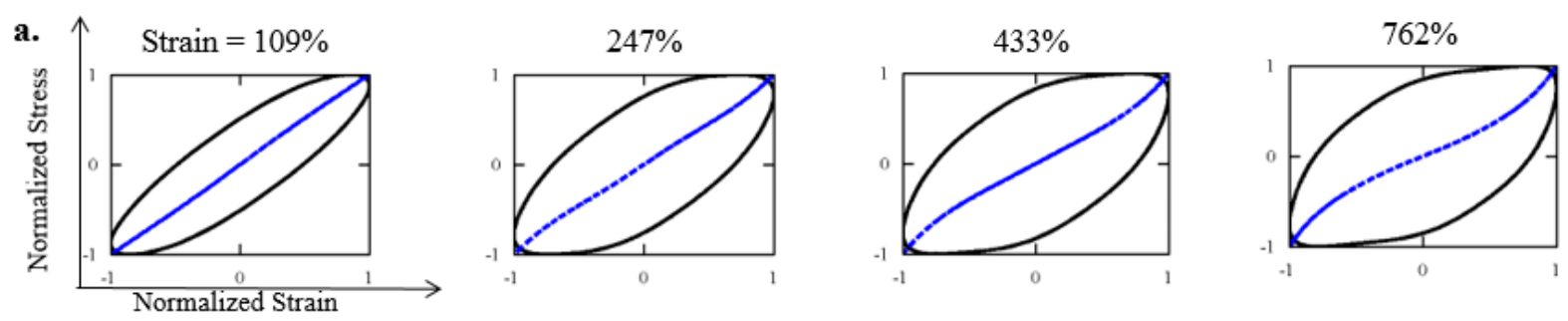

b.
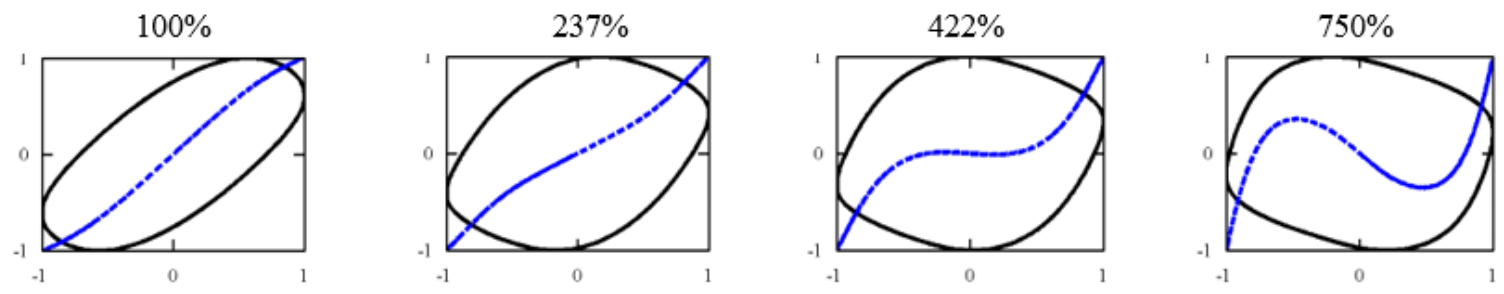

c.
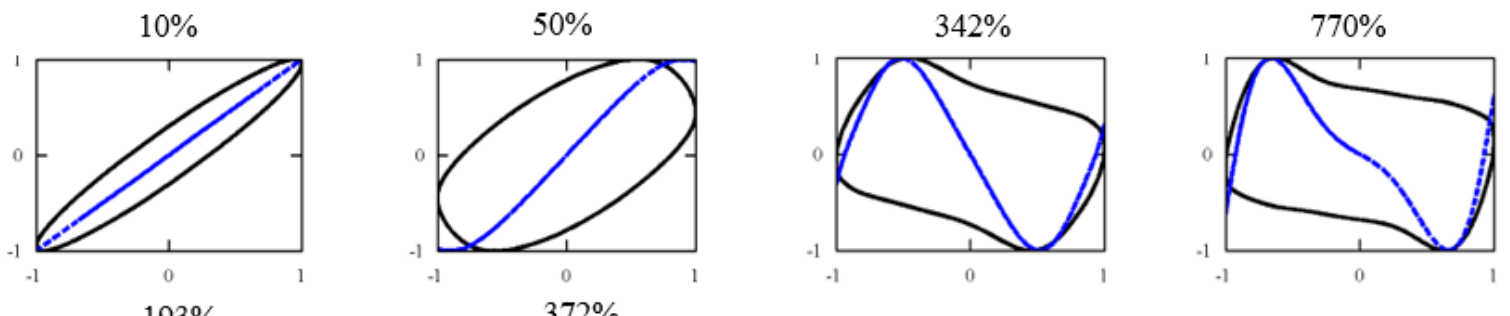

d.
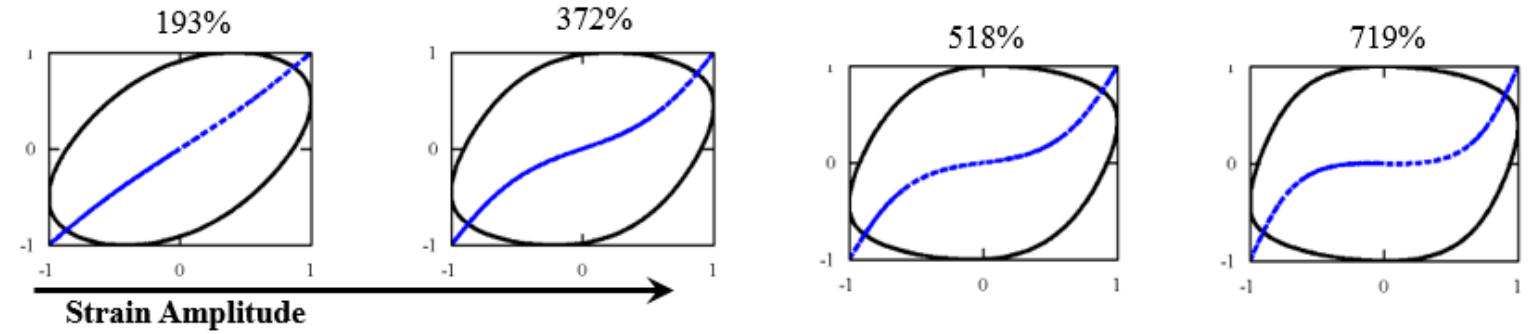

Figure 5: Elastic Lissajous-Bowditch plots showing the normalized total stress (black) and normalized elastic component of the stress (blue) versus normalized strain. The plots are shown for a series of wormlike micelles including: (a) $3 \mathrm{wt} \% 70 / 30 \mathrm{NaOA} / \mathrm{C}_{8} \mathrm{TAB}$, (b) $4.5 \mathrm{wt} \% 70 / 30 \mathrm{NaOA} / \mathrm{C}_{8} \mathrm{TAB}$, (c) $6 \mathrm{wt} \%$ $70 / 30 \mathrm{NaOA} / \mathrm{C}_{8} \mathrm{TAB}$, and (d) $6 \mathrm{wt} \% 85 / 15 \mathrm{NaOA} / \mathrm{C}_{8} \mathrm{TAB}$. 


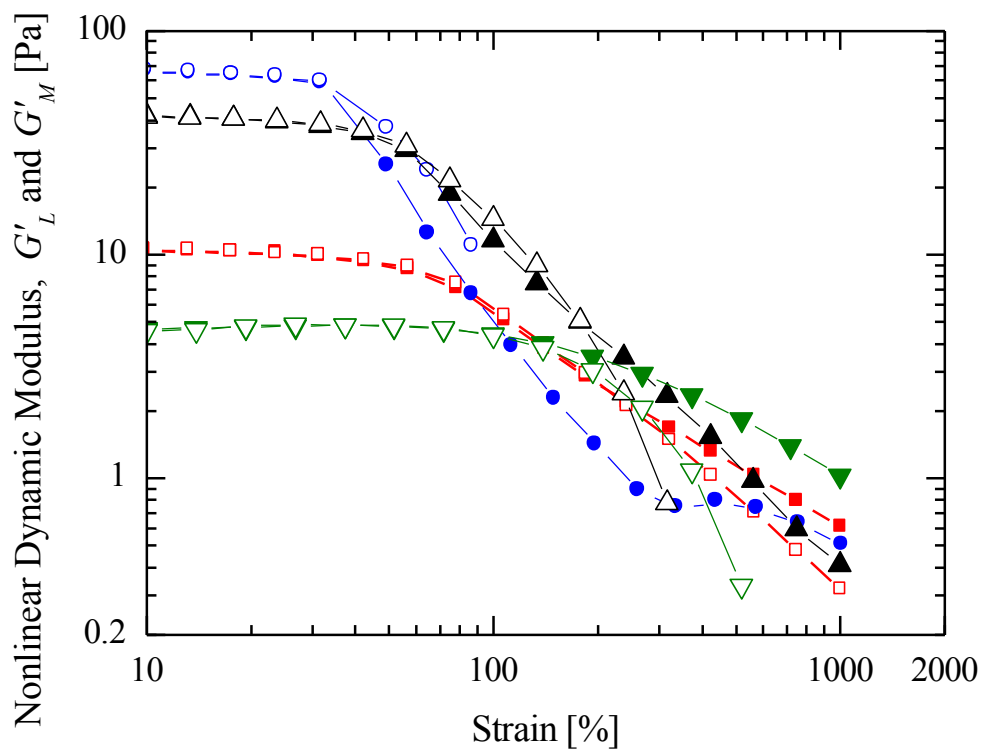

Figure 6: Nonlinear viscoelastic measurements including the large strain storage modulus, $G_{L}^{\prime}$ (solid symbols), and the minimum strain storage modulus, $G_{M}^{\prime}$ (hollow symbols), as a function of imposed strain for a series of wormlike micelle solutions including: (ロ) $3 \mathrm{wt} \% 70 / 30 \mathrm{NaOA} / \mathrm{C}_{8} \mathrm{TAB},(\boldsymbol{\Delta}) 4.5 \mathrm{wt} \%$ $70 / 30 \mathrm{NaOA} / \mathrm{C}_{8} \mathrm{TAB},(\bullet) 6 \mathrm{wt} \% 70 / 30 \mathrm{NaOA} / \mathrm{C}_{8} \mathrm{TAB}$, and $(\boldsymbol{\nabla}) 6 \mathrm{wt} \%$ 85/15 NaOA$/ \mathrm{C}_{8} \mathrm{TAB}$.

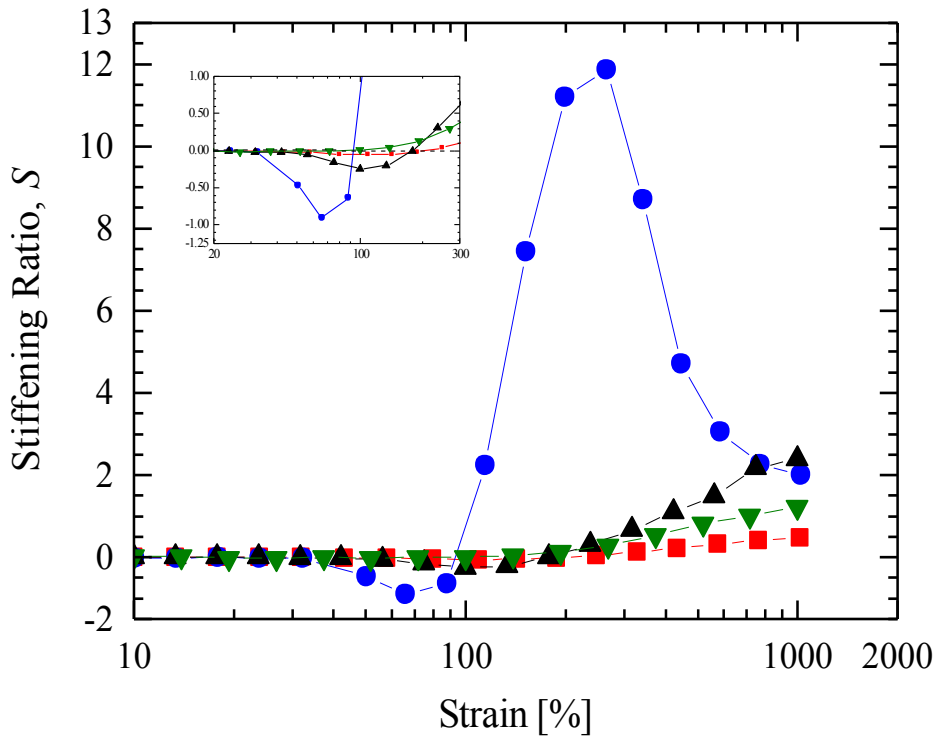

Figure 7: Stiffening Ratio, $S$, as a function of imposed strain for a series of wormlike micelle solutions including: (•) $3 \mathrm{wt} \% 70 / 30 \mathrm{NaOA} / \mathrm{C}_{8} \mathrm{TAB},(\boldsymbol{\Delta}) 4.5 \mathrm{wt} \% 70 / 30 \mathrm{NaOA} / \mathrm{C}_{8} \mathrm{TAB},(\bullet) 6 \mathrm{wt} \% 70 / 30$ $\mathrm{NaOA} / \mathrm{C}_{8} \mathrm{TAB}$, and $(\boldsymbol{\nabla}) 6 \mathrm{wt} \% 85 / 15 \mathrm{NaOA} / \mathrm{C}_{8} \mathrm{TAB}$. 


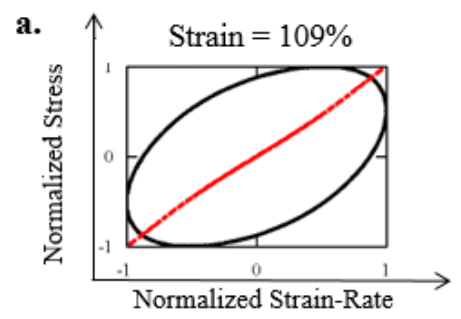

b.

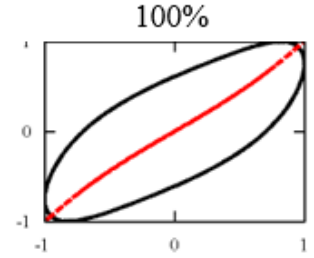

c.

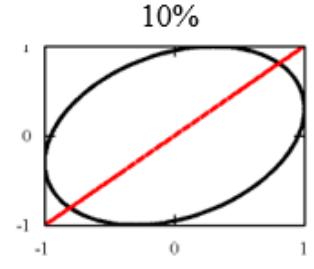

d.

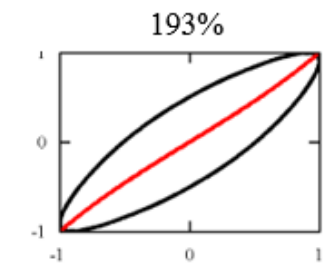

$247 \%$

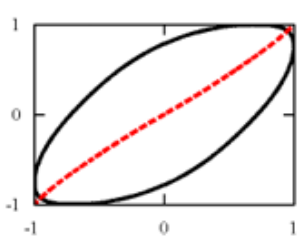

$237 \%$

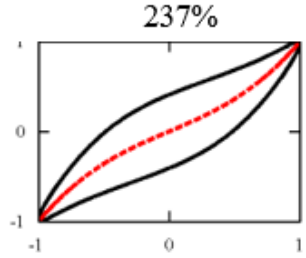

$50 \%$

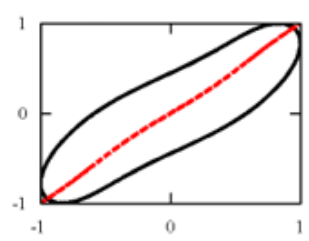

$372 \%$

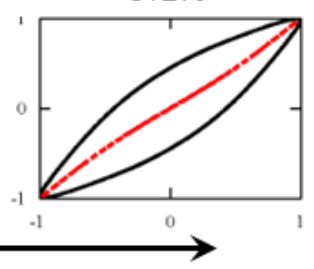

$433 \%$

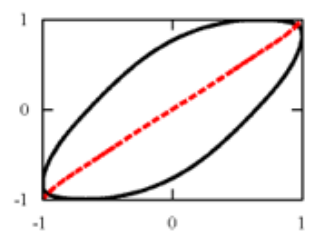

$422 \%$

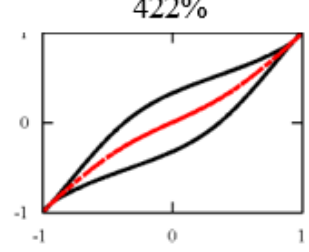

$342 \%$

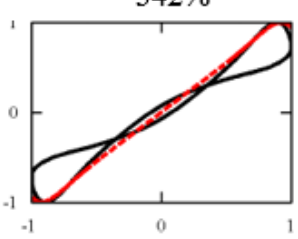

$518 \%$

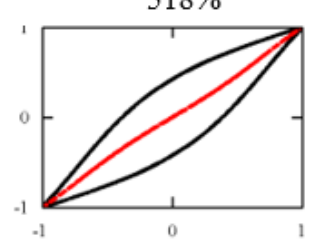

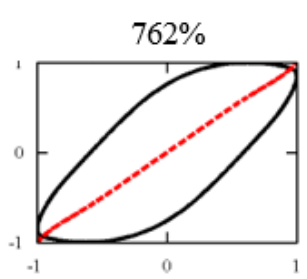

$750 \%$

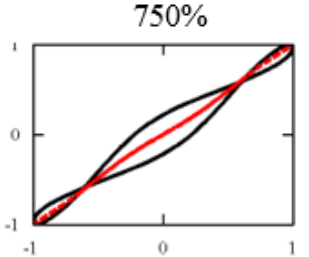

$770 \%$
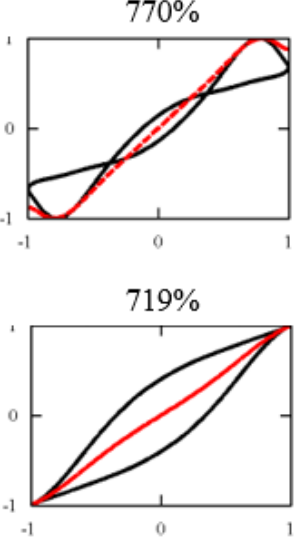

Strain Amplitude

Figure 8: Viscous Lissajous-Bowditch plots showing the normalized total stress (black) and normalized viscous component of the stress (red) versus normalized strain rate. The plots are shown for a series of wormlike micelles including: (a) $3 \mathrm{wt} \% 70 / 30 \mathrm{NaOA} / \mathrm{C}_{8} \mathrm{TAB}$, (b) $4.5 \mathrm{wt} \% 70 / 30 \mathrm{NaOA} / \mathrm{C}_{8} \mathrm{TAB}$, (c) $6 \mathrm{wt} \%$ $70 / 30 \mathrm{NaOA} / \mathrm{C}_{8} \mathrm{TAB}$, and (d) $6 \mathrm{wt} \% 85 / 15 \mathrm{NaOA} / \mathrm{C}_{8} \mathrm{TAB}$. 


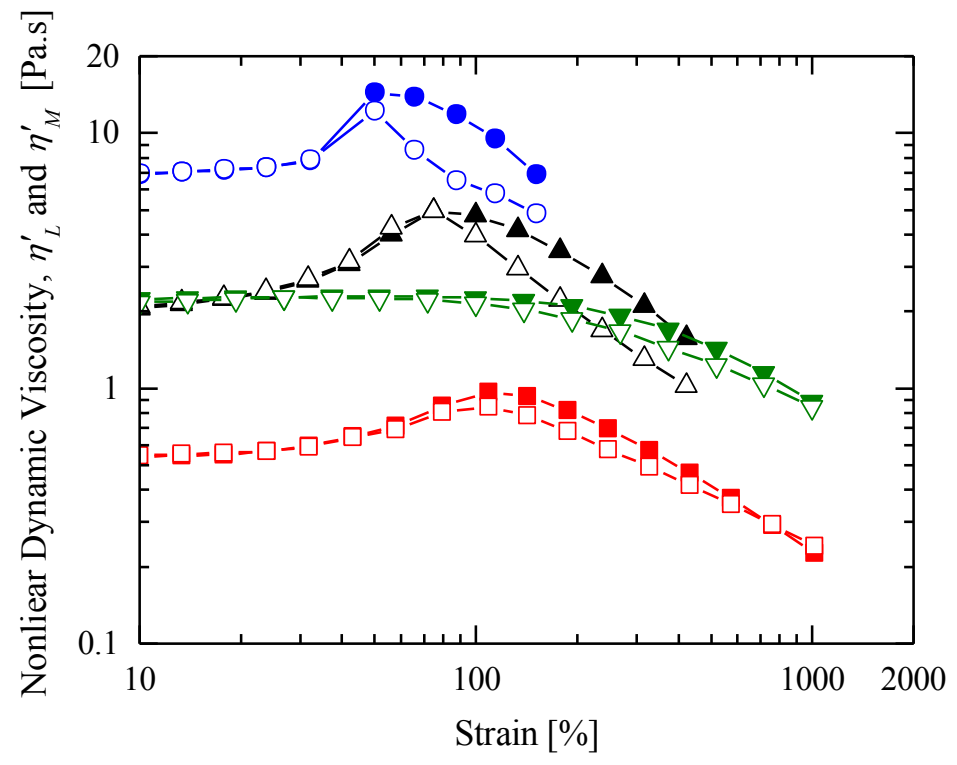

Figure 9: Nonlinear viscoelastic measurements including the large strain rate dynamic viscosity, $\eta_{L}^{\prime}$ (solid symbols), and the minimum strain rate dynamic viscosity, $\eta_{M}^{\prime}$ (hollow symbols), as a function of imposed strain for a series of wormlike micelle solutions including: (ש) $3 \mathrm{wt} \%$ 70/30 NaOA/ $\mathrm{C}_{8} \mathrm{TAB},(\boldsymbol{\Delta})$ $4.5 \mathrm{wt} \% 70 / 30 \mathrm{NaOA} / \mathrm{C}_{8} \mathrm{TAB},(\bullet) 6 \mathrm{wt} \% 70 / 30 \mathrm{NaOA} / \mathrm{C}_{8} \mathrm{TAB}$, and $(\boldsymbol{\nabla}) 6 \mathrm{wt} \% 85 / 15 \mathrm{NaOA} / \mathrm{C}_{8} \mathrm{TAB}$.

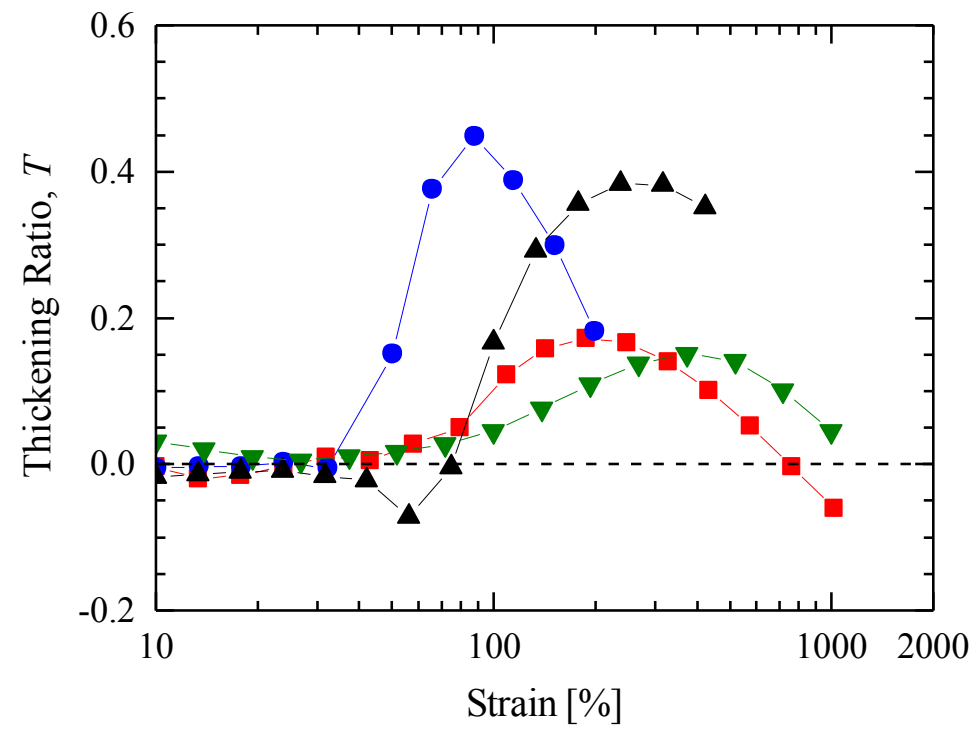

Figure 10: Thickening Ratio, $T$, as a function of imposed strain for a series of wormlike micelle solutions including: (•) 3wt $\%$ 70/30 NaOA/C $\mathrm{C}_{8} \mathrm{TAB},(\boldsymbol{\Delta}) 4.5 \mathrm{wt} \%$ 70/30 NaOA$/ \mathrm{C}_{8} \mathrm{TAB},(\bullet) 6 \mathrm{wt} \% 70 / 30$ $\mathrm{NaOA} / \mathrm{C}_{8} \mathrm{TAB}$, and $(\boldsymbol{\nabla}) 6 \mathrm{wt} \% 85 / 15 \mathrm{NaOA} / \mathrm{C}_{8} \mathrm{TAB}$. 

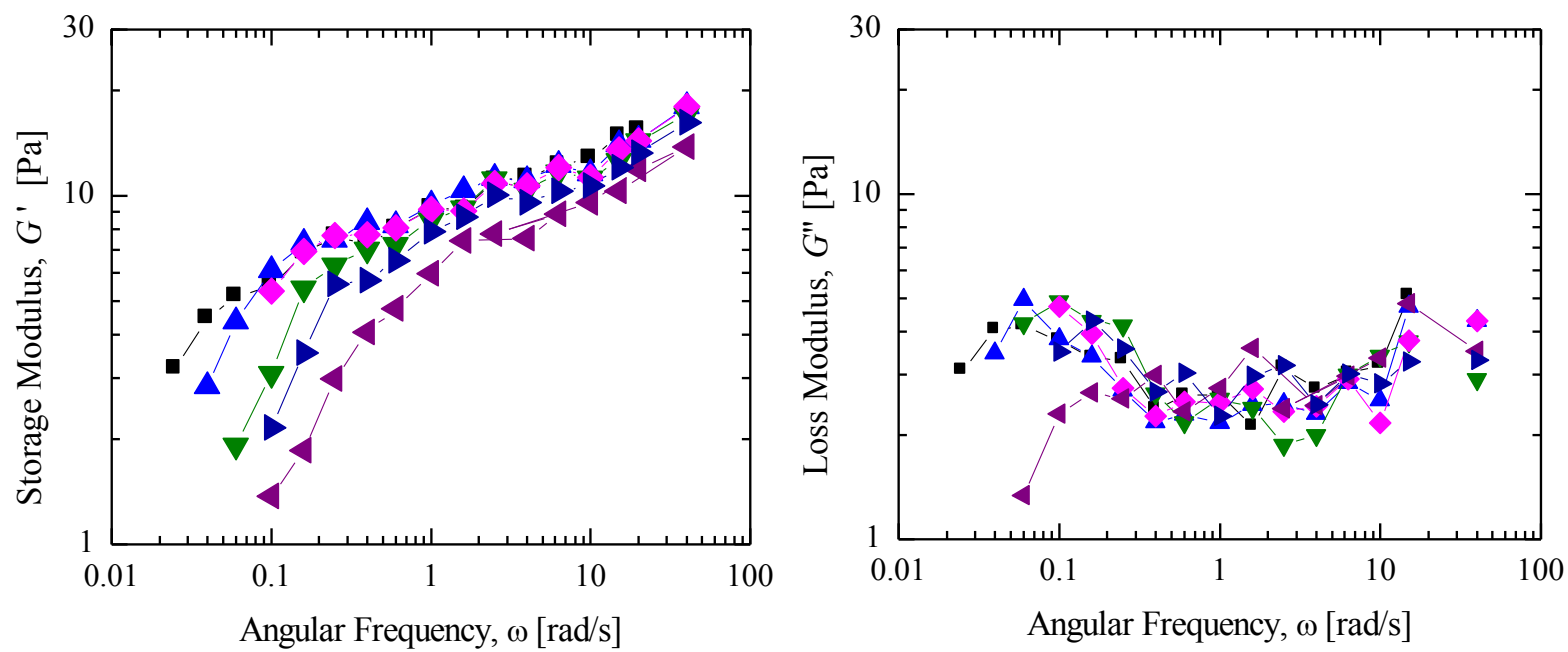

Figure 11: Orthogonal superposition measurements of storage and loss modulus for the $3 \mathrm{wt} \% 70 / 30$ $\mathrm{NaOA} / \mathrm{C}_{8} \mathrm{TAB}$ wormlike micelle solution for various applied shear rates. The data include: (ש) $\gamma=0 \mathrm{~s}^{-1}$, $(\boldsymbol{\Delta}) \gamma=0.01 \mathrm{~s}^{-1},(\diamond) \gamma=0.03 \mathrm{~s}^{-1},(\boldsymbol{\nabla}) \gamma=0.1 \mathrm{~s}^{-1},(\bullet) \gamma=0.3 \mathrm{~s}^{-1}$, and $(\bullet) \gamma=1 \mathrm{~s}^{-1}$.
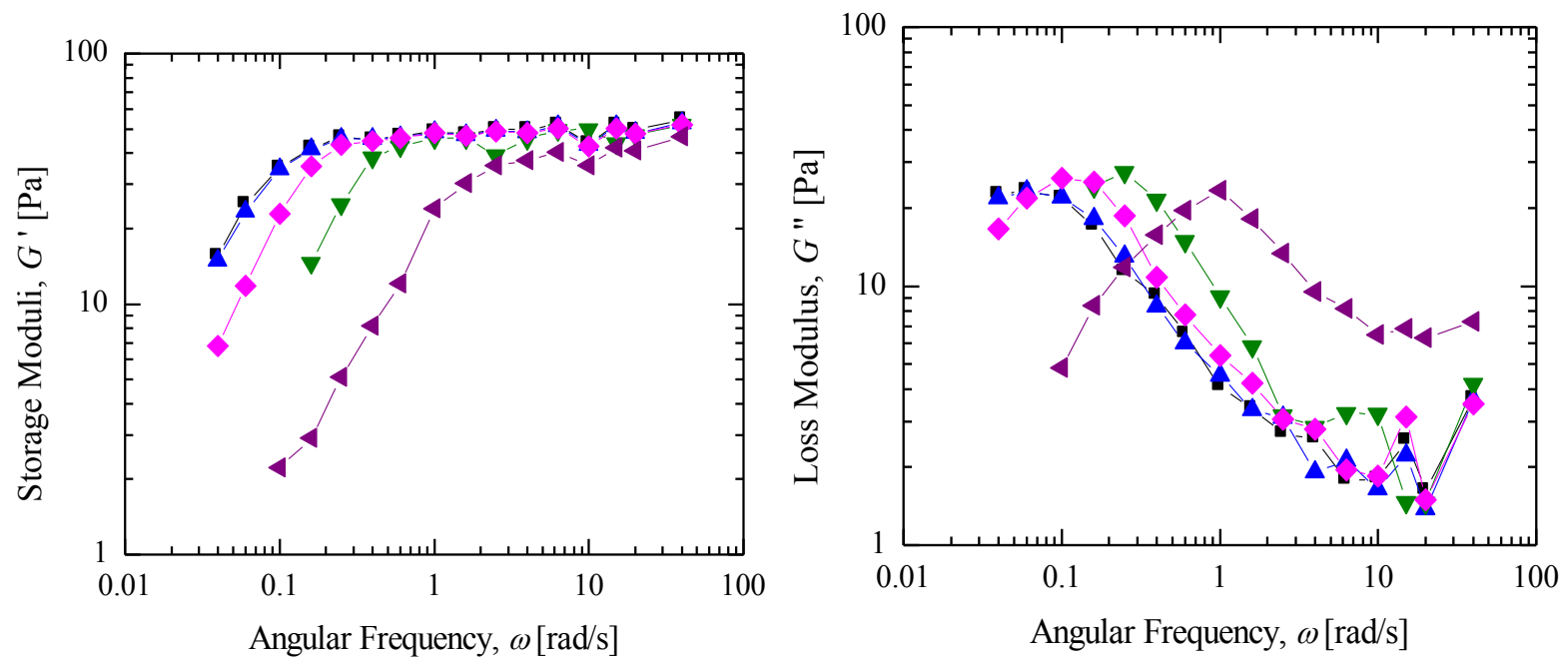

Figure 12: Orthogonal superposition measurements of storage and loss modulus for the $4.5 \mathrm{wt} \% 70 / 30$ $\mathrm{NaOA} / \mathrm{C}_{8} \mathrm{TAB}$ wormlike micelle solution for various applied shear rates. The data include: (घ) $\gamma=0 \mathrm{~s}^{-1}$, (४) $\gamma=0.01 \mathrm{~s}^{-1},(\bullet) \gamma=0.03 \mathrm{~s}^{-1},(\boldsymbol{\nabla}) \gamma=0.1 \mathrm{~s}^{-1}$ and $(\bullet) \gamma=1 \mathrm{~s}^{-1}$. 

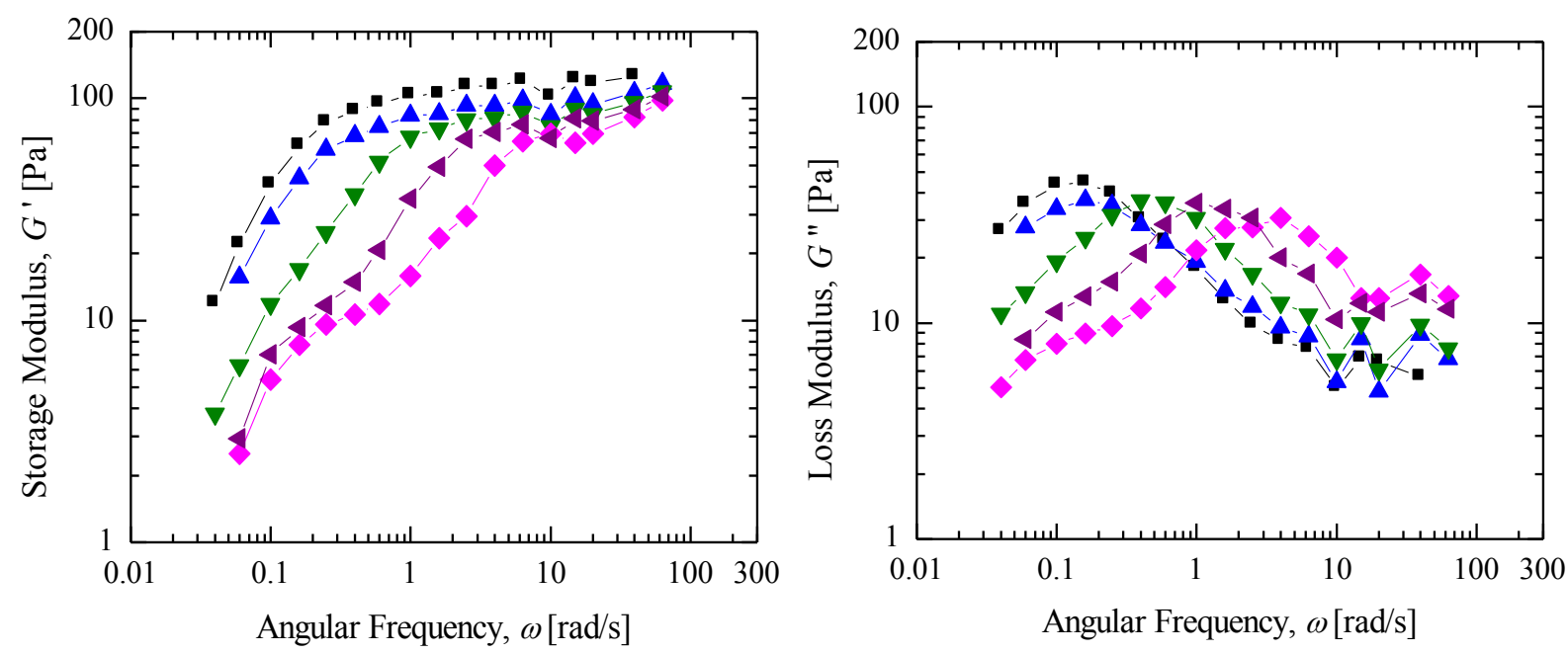

Figure 13: Orthogonal superposition measurements of storage and loss modulus for the $6 \mathrm{wt} \% 70 / 30$ $\mathrm{NaOA} / \mathrm{C}_{8} \mathrm{TAB}$ wormlike micelle solution for various applied shear rates. The data include: (घ) $\gamma=0 \mathrm{~s}^{-1}$,

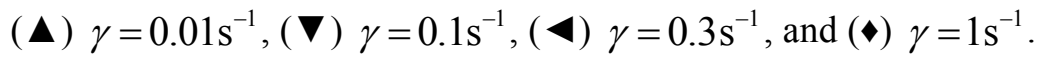
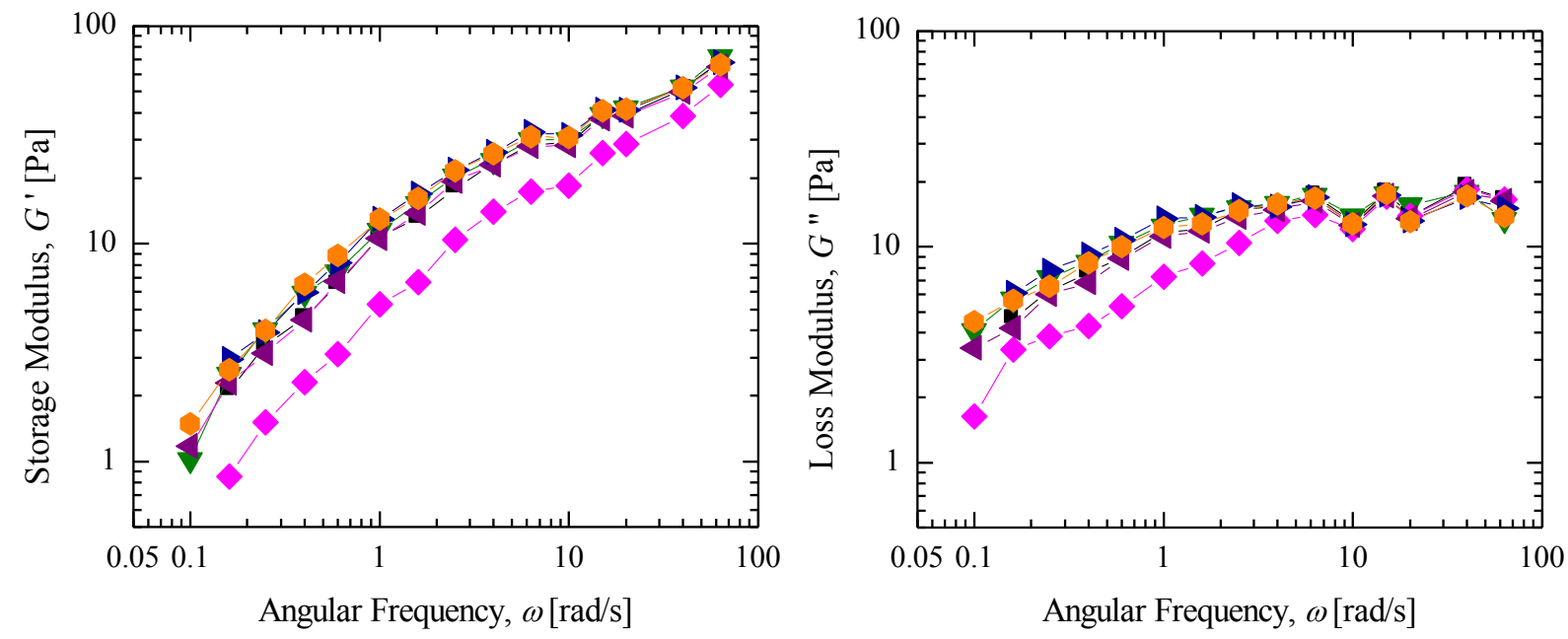

Figure 14: Orthogonal superposition measurements of storage and loss modulus for the $6 \mathrm{wt} \% 85 / 15$ $\mathrm{NaOA} / \mathrm{C}_{8} \mathrm{TAB}$ wormlike micelle solution for various applied shear rates. The data include: (घ) $\gamma=0 \mathrm{~s}^{-1}$, $(\boldsymbol{\Delta}) \gamma=0.01 \mathrm{~s}^{-1},(\boldsymbol{\nabla}) \gamma=0.1 \mathrm{~s}^{-1},(\triangleright) \gamma=0.3 \mathrm{~s}^{-1},(\mp) \gamma=0.6 \mathrm{~s}^{-1},(\triangleleft) \gamma=1 \mathrm{~s}^{-1}$, and $(\diamond) \gamma=3 \mathrm{~s}^{-1}$. 

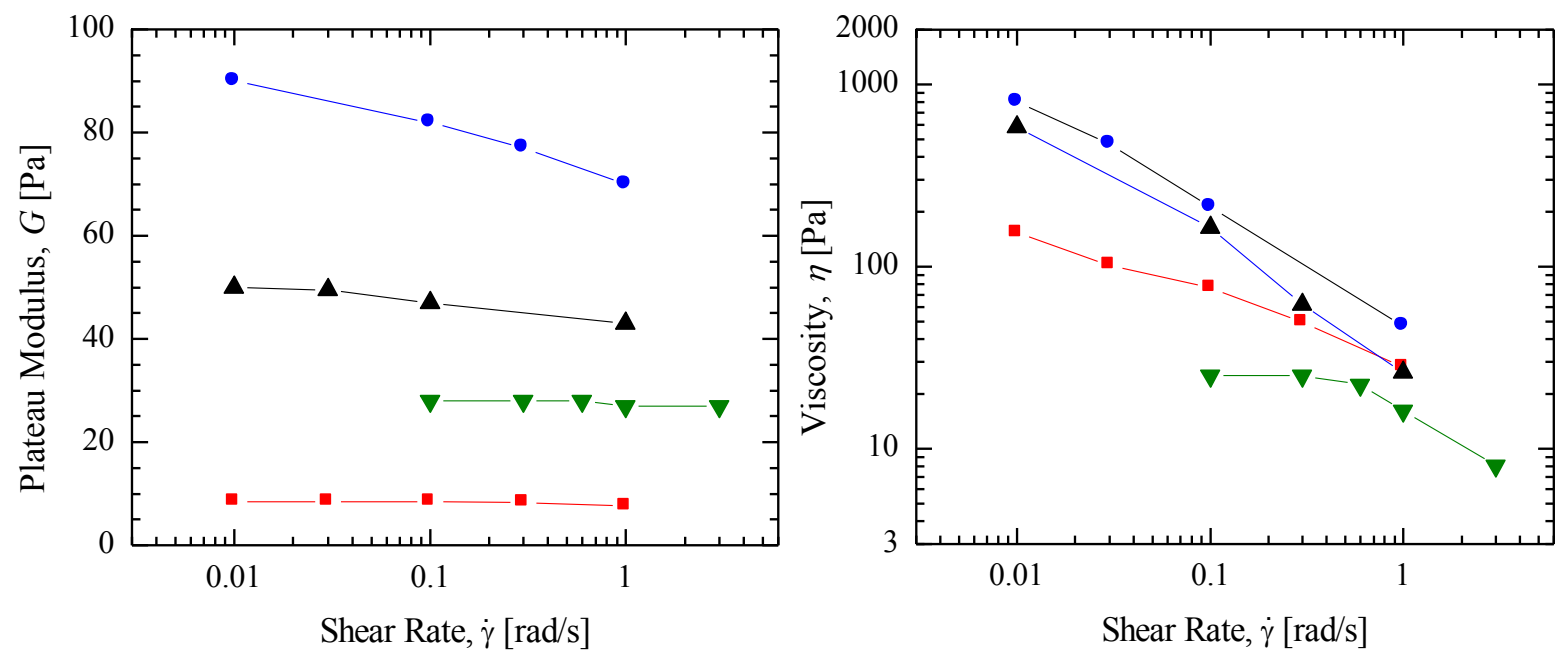

Figure 15: (a) Plateau modulus, G, and (b) viscosity as a function of shear rate for orthogonal superposition measurements of a series of wormlike micelle solutions including: (ש) $3 \mathrm{wt} \% 70 / 30$

$\mathrm{NaOA} / \mathrm{C}_{8} \mathrm{TAB},(\boldsymbol{\Delta}) 4.5 \mathrm{wt} \% 70 / 30 \mathrm{NaOA} / \mathrm{C}_{8} \mathrm{TAB},(\bullet) 6 \mathrm{wt} \% 70 / 30 \mathrm{NaOA} / \mathrm{C}_{8} \mathrm{TAB}$, and ( $)$ ) $6 \mathrm{wt} \% 85 / 15$ $\mathrm{NaOA} / \mathrm{C}_{8} \mathrm{TAB}$. The values of plateau modulus and viscosity were derived from single model Maxwell model fits to the data in Figures 11-14. 